\title{
Chemistry, Pharmacology and Therapeutic Potential of Swertiamarin - A Promising Natural Lead for New Drug Discovery and Development
}

\author{
Nur Sakinah Muhamad Fadzil \\ Mahendran Sekar (D) ' \\ Siew Hua Gan iD ${ }^{2}$ \\ Srinivasa Reddy Bonam (iD) 3 \\ Yuan Seng $\mathrm{Wu}^{4}$ \\ Jaishree Vaijanathappa ${ }^{5}$ \\ Subban Ravi ${ }^{6}$ \\ Pei Teng Lum' \\ Shivsharan B Dhadde ${ }^{7}$ \\ 'Department of Pharmaceutical \\ Chemistry, Faculty of Pharmacy and \\ Health Sciences, Universiti Kuala Lumpur \\ Royal College of Medicine Perak, Ipoh, \\ Perak, Malaysia; ${ }^{2}$ School of Pharmacy, \\ Monash University Malaysia, Bandar \\ Sunway, Selangor, Malaysia; ${ }^{3}$ Institut \\ National de la Santé et de la Recherche \\ Médicale, Centre de Recherche des \\ Cordeliers, Equipe-Immunopathologie et \\ Immunointervention Thérapeutique, \\ Sorbonne Université, Université de Paris, \\ Paris, France; ${ }^{4}$ Department of \\ Biochemistry, School of Medicine, Faculty \\ of Medicine, Bioscience and Nursing, \\ MAHSA University, Bandar Saujana Putra, \\ Selangor, Malaysia; ${ }^{5}$ Department of \\ Pharmaceutical Chemistry, JSS College of \\ Pharmacy, Mysuru, Karnataka, India; \\ ${ }^{6}$ Department of Chemistry, Karpagam \\ Academy of Higher Education, \\ Coimbatore, Tamil Nadu, India; ${ }^{7}$ D.S.T.S. \\ Mandal's College of Pharmacy, Solapur, \\ Maharashtra, India
}

Correspondence: Mahendran Sekar Department of Pharmaceutical Chemistry, Faculty of Pharmacy and Health Sciences, Universiti Kuala Lumpur Royal College of Medicine Perak, No. 3 Jalan Greentown, Ipoh, 30450, Perak, Malaysia

Tel $+6016-3346653$

Fax $+605-2536634$

Email mahendransekar@unikl.edu.my

\begin{abstract}
Swertiamarin, a seco-iridoid glycoside, is mainly found in Enicostemma littorale Blume (E. littorale) and exhibits therapeutic activities for various diseases. The present study aimed to provide a review of swertiamarin in terms of its phytochemistry, physicochemical properties, biosynthesis, pharmacology and therapeutic potential. Relevant literature was collected from several scientific databases, including PubMed, ScienceDirect, Scopus and Google Scholar, between 1990 and the present. This review included the distribution of swertiamarin in medicinal plants and its isolation, characterization, physicochemical properties and possible biosynthetic pathways. A comprehensive summary of the pharmacological activities, therapeutic potential and metabolic pathways of swertiamarin was also included after careful screening and tabulation. Based on the reported evidence, swertiamarin meets all five of Lipinski's rules for drug-like properties. Thereafter, the physicochemical properties of swertiamarin were detailed and analyzed. A simple and rapid method for isolating swertiamarin from $E$. littorale has been described. The present review proposed that swertiamarin may be biosynthesized by the mevalonate or nonmevalonate pathways, followed by the seco-iridoid pathway. It has also been found that swertiamarin is a potent compound with diverse pharmacological activities, including hepatoprotective, analgesic, anti-inflammatory, antiarthritis, antidiabetic, antioxidant, neuroprotective and gastroprotective activities. The anticancer activity of swertiamarin against different cancer cell lines has been recently reported. The underlying mechanisms of all these pharmacological effects are diverse and seem to involve the regulation of different molecular targets, including growth factors, inflammatory cytokines, protein kinases, apoptosis-related proteins, receptors and enzymes. Swertiamarin also modulates the activity of several transcription factors, and their signaling pathways in various pathological conditions are also discussed. Moreover, we have highlighted the toxicity profile, pharmacokinetics and possible structural modifications of swertiamarin. The pharmacological activities and therapeutic potential of swertiamarin have been extensively investigated. However, more advanced studies are required including clinical trials and studies on the bioavailability, permeability and administration of safe doses to offer swertiamarin as a novel candidate for future drug development.
\end{abstract}

Keywords: swertiamarin, Enicostemma littorale, biosynthesis, metabolic pathway, molecular targets, inflammatory cytokines

\section{Introduction}

Natural products obtained from medicinal plants have been investigated in recent years to improve human health by fighting a wide range of diseases. Natural products remain a source of therapeutic agents and have shown beneficial uses. 
They have a wide variety of multidimensional chemical structures; meanwhile, extensive focus has also been given to the usefulness of natural products as modifiers of biological functions. Intensive work has therefore focused on determining useful natural products for discovering novel therapeutics. $^{1,2}$

Seco-iridoid glycoside, specifically swertiamarin (Figure 1), has received extensive interest in recent years due to its abundant occurrence in medicinal plants and the fact that it possesses many beneficial pharmacological properties. It is mainly obtained from Enicostemma species that belong to the Gentianaceae family. Compared to other seco-iridoid glycosides isolated from medicinal plants, swertiamarin has been found to possess multifunctional properties, including analgesic, anti-inflammatory, antiarthritis, hepatoprotective, antidiabetic, antioxidant, antimicrobial, anticancer, neuroprotective and gastroprotective properties. Swertiamarin modulates a wide range of molecular targets, including inflammatory cytokines, transcription factors, protein kinases, growth factors, apoptosis-related proteins, receptors, and enzymes and their signaling pathways, in various pathological conditions. Nevertheless, despite conferring numerous beneficial effects, there is no detailed and comprehensive review of swertiamarin to date.

Therefore, in this review, we aimed to conduct a systematic review of swertiamarin with respect to its

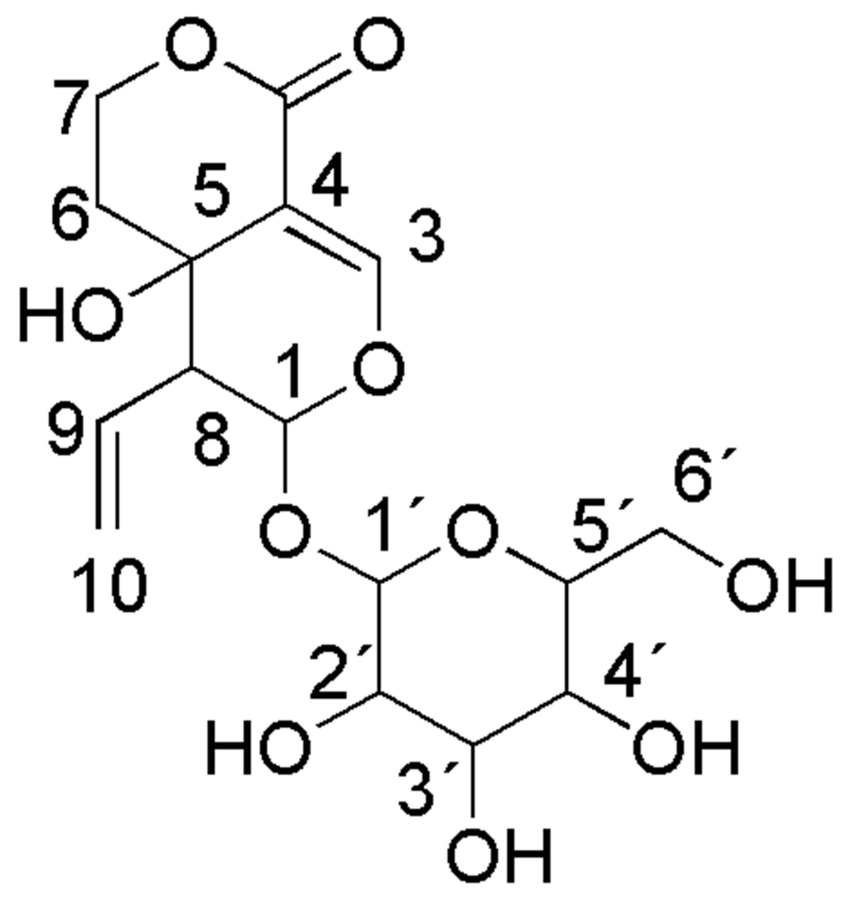

Figure I Chemical structure of swertiamarin. phytochemical, physicochemical, pharmacological and therapeutic potential, including the cellular and molecular mechanisms of action, and to provide possible biosynthetic pathways.

\section{Methods}

Relevant literature was collected from several scientific databases, including PubMed, ScienceDirect, Scopus and Google Scholar. The literature search of the scientific evidence of swertiamarin published since 1990 was achieved using the following keywords: "Swertiamarin" OR "Enicostemma littorale" OR "Enicostemma axillare" OR "Iridoids" OR "Secoiridoids" AND "Chemistry" OR "Biosynthesis" OR "in vitro" OR "in vivo" OR "Biological studies" OR "Pharmacological studies" OR "Molecular mechanisms" OR "Gene expressions" OR "Toxicity studies" OR "Clinical studies" OR "Pharmacokinetics" OR "Pharmacodynamics". Studies that were not written in English and did not have any abstracts were excluded from the initial screening. There was no restriction to be followed for collecting the studies carried out on swertiamarin, especially regarding the aspects of dose, route of administration, duration of treatment, or animal versus human studies. The articles were chosen for the final analysis after applying the inclusion and exclusion criteria and removing duplicates from the databases (Figure 2). The information included in the review was divided into two main categories: phytochemistry and pharmacological properties of swertiamarin. They were further categorized according to the key findings of the study (Figure 3, Figure 4, Figure 5, Figure 6). After a complete screening, the obtained information was summarized and included in the present review. In addition, this review elaborates the molecular mechanisms involved in its pharmacological actions.

\section{Swertiamarin and Its Phytochemistry Origin and Distribution of Swertiamarin}

Collectively, swertiamarin has been identified and found to be abundant in Enicostemma species (E. littorale) ${ }^{3}$ This tropical genus is widespread throughout Asia, South America and Africa. ${ }^{4}$ E. littorale grows in savannas, grasslands, forests and beaches, from wet to very dry climates in many different environments, and it even lives in a very saline climate. ${ }^{4}$ Swertiamarin is a key component of many crude drugs that are sold in Japan and other countries, and their high swertiamarin content is usually responsible for the effects of these crude drugs. ${ }^{5,6}$

Apart from E. littorale, swertiamarin also presents in many other plant species, including Centaurium 


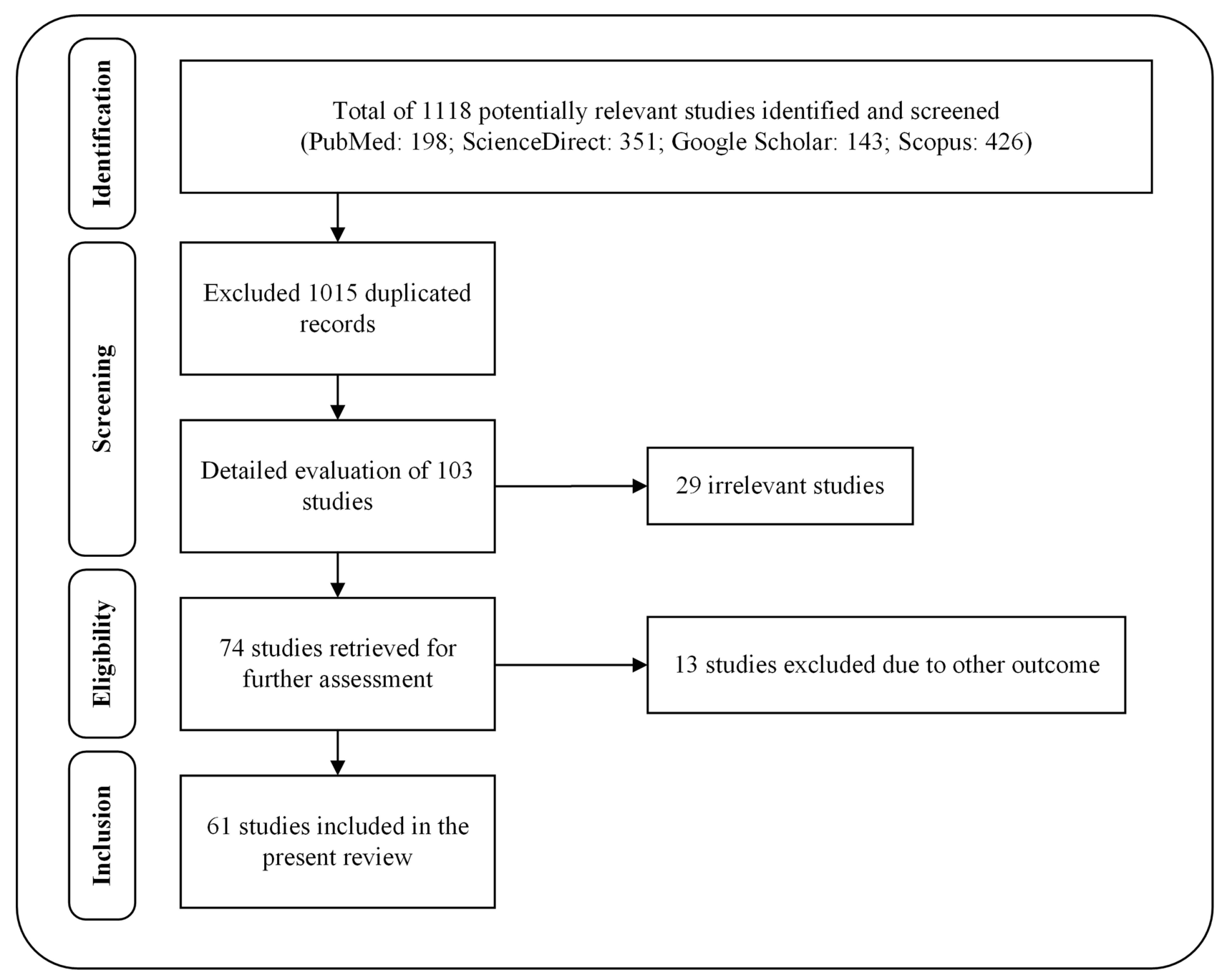

Figure 2 Flow chart of the stages of inclusion of studies in the review.

erythraea, ${ }^{7}$ Eustoma grandiflorum, ${ }^{8}$ Fragrea fragrans, ${ }^{9}$ Gentiana algida, ${ }^{10}$ Gentiana apiata, ${ }^{11}$ Gentiana asclepiadea, ${ }^{12}$ Gentiana crassicaulis $^{13}$ Gentiana cruciata $^{14}$ Gentiana dahurica, ${ }^{15}$ Gentiana davidii, ${ }^{16}$ Gentiana gelida, ${ }^{17}$ Gentiana kurroo, ${ }^{18}$ Gentiana lawrencei, ${ }^{19}$ Gentiana lhassica, ${ }^{15}$ Gentiana lutea, ${ }^{20}$ Gentiana macrophylla, ${ }^{15}$ Gentiana manshurica, ${ }^{21}$ Gentiana rigescens, ${ }^{22}$ Gentiana robusta, Gentiana straminea, Gentiana tibetica, Gentiana waltonii, ${ }^{15}$ Genus swertia $^{23}$ Swertia angustifolia, ${ }^{24}$ Swertia chirayita, ${ }^{25}$ Swertia corymbose, ${ }^{26}$ Swertia delavayi, ${ }^{27}$ Swertia franchentiana $^{28}$ Swertia hispidicalyx, ${ }^{29}$ Swertia japonica, ${ }^{30}$ Swertia kouitchensis, ${ }^{31}$ Swertia longifolia, ${ }^{32}$
Swertia mileensis, ${ }^{33}$ Swertia mussotii, ${ }^{34}$ Swertia patens, ${ }^{35}$ Swertia psudochinesis, ${ }^{36}$ and Swertia punicea. ${ }^{37}$

\section{Medicinal Uses of $E$. littorale}

In Ayurveda, E. littorale Blume is known as "Nagajihva" or "Mamejawa". It is an evergreen herb generally found in the Indian and Tropical African coastal regions. Traditionally, the plant is used for the treatment of diabetes, rheumatism, stomach ulcers, swelling and insect poisoning. Furthermore, antipyretic, antimalarial, anti-inflammatory, antidiabetic and antimicrobial activities have also been demonstrated. ${ }^{38}$ Various parts of E. littorale have been used for specific purposes. For example, roots are used to treat ailments, 


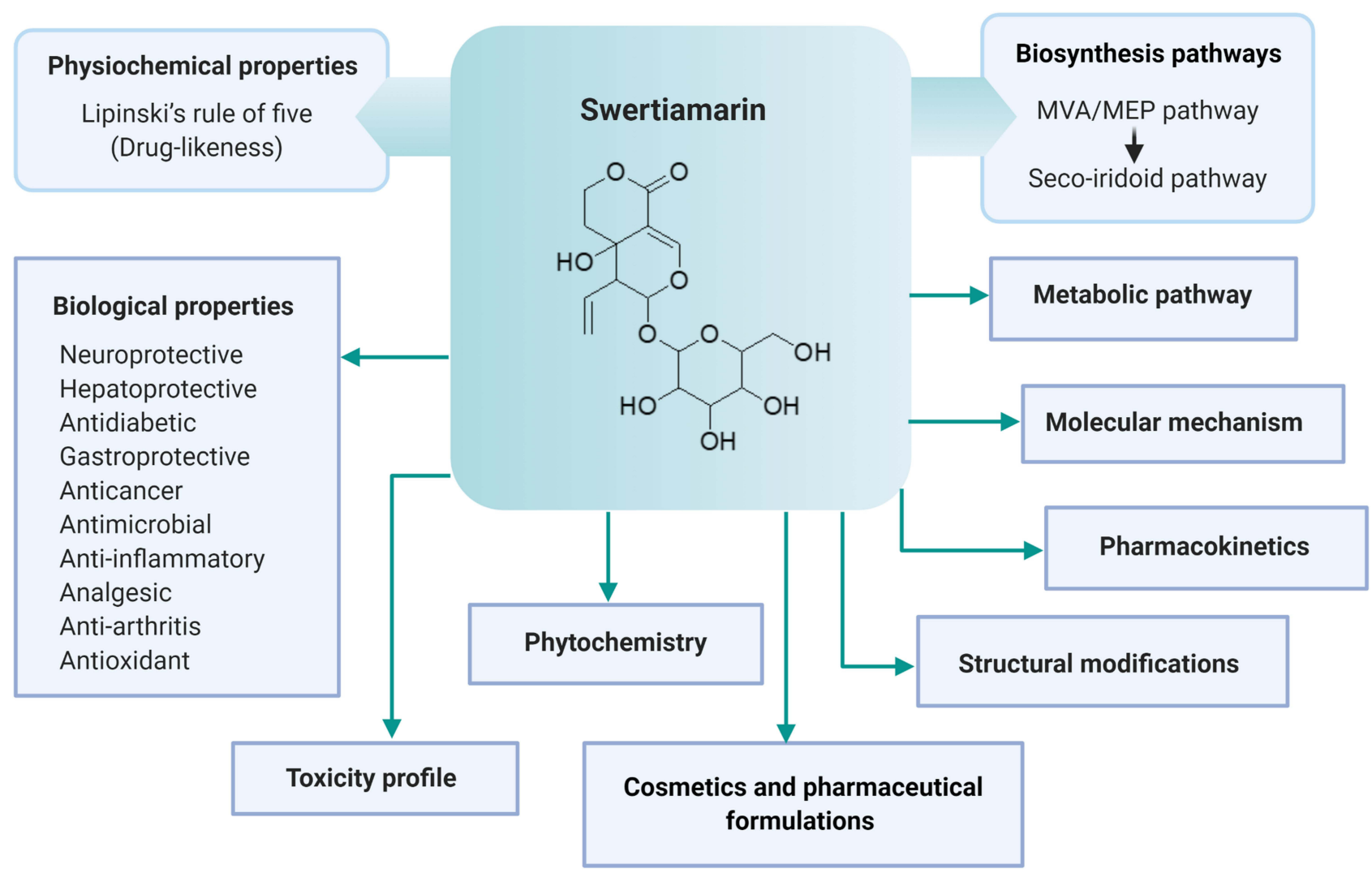

Figure 3 Key findings of swertiamarin included in the review.

including malaria, skin diseases, leprosy and diabetes. Leaves are reported to possess protective effects, such as hypoglycemic, antioxidant, hepatoprotective and hepatomodulatory properties, and to ameliorate obesity. ${ }^{39}$

\section{Biochemistry of Swertiamarin}

Plants belonging to the family Gentianaceae are known for their bitter taste, which is supported by many compounds, including swertiamarin. ${ }^{40} \mathrm{~A}$ biochemical pathway that converts swertiamarin to erythrocentaurin has been suggested. Hydrolysis of swertiamarin is facilitated by the bacterial $\beta$ glucosidase enzyme to produce an aglycone. Subsequently, it was discovered that in vivo, the metabolism of the monoterpene compound swertiamarin to dihydroisocoumarin (an alkaloid) might be the main contributing structure that confers its pharmacological effect. ${ }^{15}$

\section{Isolation of Swertiamarin}

Jaishree et $\mathrm{al}^{41}$ reported the conventional isolation method of swertiamarin from the ethyl acetate extract of Enicostemma littorale (E. littorale). Briefly, the powdered whole plant of E. axillare $(250 \mathrm{~g})$ was successively extracted with petroleum ether, chloroform, ethyl acetate and methanol (1.5 liters each) in a Soxhlet extractor for 18-20 h. Subsequently, the extracts were concentrated and dried under reduced pressure and a controlled temperature. The extract, which yielded a brown semisolid, was chromatographed with silica gel and then eluted with 1) ethyl acetate, 2) ethyl acetate: acetone and 3) acetone mixtures. The ethyl acetate: acetone fractions were collected and rechromatographed (silica gel, 100-200 mesh). The elution was conducted using chloroform: methanol (9:1), which gave pure swertiamarin (yield $1 \mathrm{~g}, 0.4 \%$ ). However, a rapid method for isolating swertiamarin from $E$. littorale has been found to have an almost five times higher yield ${ }^{42}$ than the conventional method reported by Jaishree et al. ${ }^{41}$ In brief, 25 $\mathrm{g}$ of powdered plant material was defatted with petroleum ether followed by extraction with methanol $(4 \times 200 \mathrm{~mL})$ by cold maceration. Approximately $50 \mathrm{~mL}$ of the methanolic extract was obtained after purification and concentration under vacuum. The extract was then treated with cold diethyl ether to produce a precipitate $(8.66 \mathrm{~g})$. A fraction of the precipitate ( $3 \mathrm{~g}$ ) on silica gel was chromatographed (by column chromatography) and eluted with petroleum ether. The elution obtained included 1) petroleum ether containing ethyl acetate $(0-18 \%), 2)$ ethyl acetate and 3 ) ethyl acetate containing increasing quantities of methanol (0-12\%). Thin layer chromatography (TLC) was used to track the different 
fractions obtained using ethyl acetate: methanol: water (7.7:1.5:0.5). Thereafter, the fractions containing swertiamarin were combined and concentrated to dryness (yield 0.53 g, $2.12 \%)$.

\section{Structural Characterization of Swertiamarin}

A very detailed structural characterization of swertiamarin is given below based on the values obtained from spectroscopic methods, including UV, FT-IR, ${ }^{1} \mathrm{H}$ NMR, ${ }^{13} \mathrm{C}$ NMR and ESI-MS. The obtained values are comparable with the spectral data published earlier by other researchers. ${ }^{43,44}$

UV ( $\lambda$ max): $236.8 \mathrm{~nm}$ in methanol.

FT-IR (KBr): $3450 \mathrm{~cm}^{-1}(-\mathrm{OH}), 1750 \mathrm{~cm}^{-1}(\mathrm{C}=\mathrm{O})$, $1650 \mathrm{~cm}^{-1}(\mathrm{C}=\mathrm{C})$ and 1390 to $860 \mathrm{~cm}^{-1}(\mathrm{C}-\mathrm{O})$.

${ }^{1} \mathrm{H}$ NMR (DMSO, $400 \mathrm{MHz}$ ): Swertiamarin showed vinyl group protons at $\delta 5.30(1 \mathrm{H}, \mathrm{dd}, 9.2 \mathrm{~Hz}, 2.5 \mathrm{~Hz}$, $\mathrm{H}-10 \alpha), 5.36(1 \mathrm{H}, \mathrm{dd}, 16.3 \mathrm{~Hz}, 2.5 \mathrm{~Hz}, \mathrm{H}-10 \beta)$ and at $\delta$ $5.42(1 \mathrm{H}, \mathrm{m}, \mathrm{H}-8)$, acetal methine proton at $\delta 5.72(1 \mathrm{H}, \mathrm{d}, 2$ $\mathrm{Hz}, \mathrm{H}-1)$ and a trisubstituted double bond at $\delta 7.63(1 \mathrm{H}, \mathrm{s}$, $\mathrm{H}-3)$. An anomeric proton signal at $\delta 4.63\left(1 \mathrm{H}, \mathrm{d}, 7 \mathrm{~Hz}, \mathrm{H}-1^{\prime}\right)$ was also recognized. Additional signals corresponding to the glucopyranocyl group appeared at $\delta 3.20(1 \mathrm{H}, \mathrm{dd}, 8.5 \mathrm{~Hz}, 8.1$ Hz, H-2'), 3.32 (2H, m, H-3', H-4'), 3.65 (2H, dd, 8.8 Hz, 8.1 $\left.\mathrm{Hz}, \mathrm{H}-5^{\prime}, \mathrm{H}-6^{\prime} \alpha\right)$ and $3.89\left(1 \mathrm{H}, \mathrm{dd}, 8.5 \mathrm{~Hz}, 8.1 \mathrm{~Hz}, \mathrm{H}-6^{\prime} \beta\right)$. The signals at $\delta 4.33(1 \mathrm{H}$, ddd, $11.0 \mathrm{~Hz}, 5.1 \mathrm{~Hz}, 3.3 \mathrm{~Hz}$, $\mathrm{H}-7 \alpha$ ) and $4.75(1 \mathrm{H}, \mathrm{ddd}, 13.5 \mathrm{~Hz}, 11.0 \mathrm{~Hz}, 2.9 \mathrm{~Hz}, \mathrm{H}-7 \beta)$ are due to an oxymethylene group. The signals at $\delta 1.75(1 \mathrm{H}, \mathrm{d}$, $14.0 \mathrm{~Hz}, \mathrm{H}-6 \alpha)$ and $1.90(1 \mathrm{H}, \mathrm{ddd}, 13.9 \mathrm{~Hz}, 13.2 \mathrm{~Hz}, 5.1 \mathrm{~Hz}$, $\mathrm{H}-6 \beta$ ) are attributed to a methylene group and the other lone signal at $\delta 2.91(1 \mathrm{H}, \mathrm{dd}, 9.2 \mathrm{~Hz}, 1.5 \mathrm{~Hz} \mathrm{H}-9)$ corresponds to a methine proton.

${ }^{13} \mathrm{C}$ NMR (DMSO, $100 \mathrm{MHz}$ ): Swertiamarin displayed 16 signals in the ${ }^{13} \mathrm{C}$-NMR spectrum. Out of these signals, six signals were assigned to the glucose moiety. The signal at $\delta 100.2$ is attributed to the anomeric carbon of the glucose moiety, and the $-\mathrm{CH}_{2} \mathrm{OH}$ carbon appeared at $\delta 62.6\left(\mathrm{C}-6^{\prime}\right)$. The other four carbons under oxygen functionality in the glucose moiety appeared at $\delta 74.4$ (C-2'), 77.8 (C-3'), 71.4 $\left(\mathrm{C}-4^{\prime}\right)$ and $78.6\left(\mathrm{C}-5^{\prime}\right)$. With respect to the aglycone portion of this compound, the signal at $\delta 99.1$ is the characteristic signal of the $\mathrm{C}-1$ atom of the seco-iridoid moiety. The signals at $\delta 121.2(\mathrm{C}-10)$ and $133.8(\mathrm{C}-8)$ are attributed to the vinylic portion of the compound. The carbonyl group appeared at $\delta$ 167.9. The signals at $\delta 154.7(\mathrm{C}-3)$ and 108.9 (C-4) were assigned to the double bond between $\mathrm{C}-3$ and C-4. The signals in the upfield region at $\delta 64.3$ and 65.9 are due to carbon atoms in the oxygen functional groups at C-5 and C-7, respectively, and the signal at $\delta 33.8$ is due to a methylene carbon atom at C-6.

ESI-MS (m/z): $375[\mathrm{M}+1], 397\left[\mathrm{M}+\mathrm{Na}^{+}\right]$and $413[\mathrm{M}$ $\left.+\mathrm{K}^{+}\right]$. The molecular weight of swertiamarin is 374 with a molecular formula of $\mathrm{C}_{16} \mathrm{H}_{22} \mathrm{O}_{10}$.

\section{Physicochemical Properties of Swertiamarin}

There are several barriers to overcome for any drug molecule to enter into its active site. However, many complicated biological processes can be modeled using simple physical chemistry models or properties, and understanding these often drives both the lead optimization and lead identification phases of a drug discovery program forward. There are certain physicochemical properties that allow a molecule to become a drug-like molecule: the molecule should be 1) small enough to be transported throughout the body, 2) hydrophilic enough to dissolve in the bloodstream, 3) lipophilic enough to cross fat barriers within the body, and 4) contain enough polar groups to enable it to bind to a receptor, but not so many that it would be eliminated to quickly from the body via the urine before exerting therapeutic effects. The physicochemical properties of swertiamarin were mainly obtained from PubChem $^{45}$ and other trustworthy databases. The druglike properties (molecular weight, H-bond donors, H-bond acceptors, $\log P$ value and rotatable bonds) as mentioned in Lipinski's rule of five ${ }^{46}$ were determined by using Biovia Discovery studio version 19.0. A drug molecule possesses one or more functional groups positioned in three-dimensional space on a structural framework that holds functional groups in a defined geometrical array that enables the molecule to bind specifically to a targeted biological macromolecule, the receptor. All the obtained details about the physicochemical properties of swertiamarin are summarized in Table 1.

\section{Biosynthesis of Swertiamarin}

The biosynthetic pathway of seco-iridoid is mostly unresolved. It begins with geraniol and is thought to consist of approximately 10 enzymes that catalyze successive reactions of oxidation, reduction, glycosylation and methylation. ${ }^{47}$ Only the first step (geraniol 10-hydroxylase, G-10H) and the last two steps (loganic acid O-methyltransferase, LAMT; and secologanin synthase, SLS) are well known, although the pathway has been explored for many years. ${ }^{47-52}$ Isopentenyl pyrophosphate (IPP) is an essential intermediate in the biosynthesis of terpenes 
Table I Computed Physicochemical Properties of Swertiamarin

\begin{tabular}{|c|c|}
\hline Property & Result/value \\
\hline Common name & Swertiamarin \\
\hline Synonyms & Swertiamarine; Swertiamaroside; Swertiamari; Wertiamarin \\
\hline Category & Iridoid/Seco-iridoid \\
\hline IUPAC name & $\begin{array}{l}(3 S, 4 R, 4 a R) \text {-4-ethenyl-4a-hydroxy-3-[(2S,3R,4S,5S,6R)-3,4,5-trihydroxy-6-(hydroxymethyl)oxan-2-yl]oxy- } \\
\text { 3,4,5,6-tetrahydropyrano[3,4-c]pyran-8-one }\end{array}$ \\
\hline Other name(s) & $\begin{array}{l}\text { (4aR,5R,6S)-5-ethenyl-6-( } \beta \text {-D-glucopyranosyloxy)-4,4a,5,6-tetrahydro-4a-hydroxy-IH,3H-pyrano[3,4-c]pyran-I-one; } \\
\text { 4,4a,5,6-tetrahydro-4aa-hydroxy-I-oxo-5b-vinyl- IH,3H-pyrano[3,4-c]pyran-6-yl b-D-glucopyranoside; } \\
\text { 4,4a,5,6-tetrahydro-4a-hydroxy-6-(tetrahydro-3,4,5-trihydroxy-6-(hydroxymethyl)-2H-pyran-2-yloxy)-5-vinylpyrano } \\
\text { [3,4-c]pyran-(3H)-one }\end{array}$ \\
\hline Canonical SMILES & $\mathrm{C}=\mathrm{CCIC}(\mathrm{OC}=\mathrm{C} 2 \mathrm{Cl}(\mathrm{CCOC} 2=0) \mathrm{O}) \mathrm{OC} 3 \mathrm{C}(\mathrm{C}(\mathrm{C}(\mathrm{C}(\mathrm{O} 3) \mathrm{CO}) \mathrm{O}) \mathrm{O}) \mathrm{O}$ \\
\hline Isomeric SMILES & $\begin{array}{l}\mathrm{C}=\mathrm{C}[\mathrm{C} @ \mathrm{H}] \mathrm{I}[\mathrm{C} @ \mathrm{H}](\mathrm{OC}=\mathrm{C} 2[\mathrm{C} @] \mathrm{I}(\mathrm{CCOC}=\mathrm{O}) \mathrm{O}) \mathrm{O}[\mathrm{C} @ \mathrm{H}] 3[\mathrm{C} @ @ \mathrm{H}]([\mathrm{C} @ \mathrm{H}]([\mathrm{C} @ @ \mathrm{H}]([\mathrm{C} @ \mathrm{H}](\mathrm{O}) \mathrm{CO}) \\
\text { O)O)O }\end{array}$ \\
\hline Molecular formula & $\mathrm{C}_{16} \mathrm{H}_{22} \mathrm{O}_{10}$ \\
\hline Molecular weight & $374.34 \mathrm{~g} / \mathrm{mol}$ \\
\hline Hydrogen bond donors & 5 \\
\hline Hydrogen bond acceptors & 10 \\
\hline Rotatable bonds & 4 \\
\hline $\begin{array}{l}\log P \text { (partition coefficient } \\
\text { value) }\end{array}$ & 0.64 (Predicted) \\
\hline Molar refractivity & $82.12 \mathrm{~cm}^{3}$ \\
\hline $\begin{array}{l}\text { Topological polar surface } \\
\text { area }\end{array}$ & $155 \AA^{2}$ \\
\hline Percent composition & $\mathrm{C}=51.34 \%, \mathrm{H}=5.92 \%, \mathrm{O}=42.74 \%$ \\
\hline Optical rotation & {$[\alpha] \mathrm{D} 20-127^{\circ}(\mathrm{c}=\mathrm{l}$ in $96 \%$ ethanol $)$} \\
\hline XLogP3-AA & -2 \\
\hline Molar mass & $374.121297 \mathrm{Da}$ \\
\hline Monoisotopic mass & $374.121297 \mathrm{Da}$ \\
\hline Heavy atom count & 26 \\
\hline Formal charge & 0 \\
\hline Complexity & 592 \\
\hline Isotope atom count & 0 \\
\hline Atom stereocenter count & 8 \\
\hline Bond stereocenter count & 0 \\
\hline $\begin{array}{l}\text { Covalently-bonded unit } \\
\text { count }\end{array}$ & 1 \\
\hline Canonicalized & Yes \\
\hline
\end{tabular}

(Continued) 
Table I (Continued).

\begin{tabular}{|l|l|}
\hline Property & Result/value \\
\hline Melting point & $\mathrm{II} 3-1 \mathrm{I} 4{ }^{\circ} \mathrm{C}$ \\
\hline Boiling point & $649.3 \pm 55.0^{\circ} \mathrm{C}$ (Predicted) \\
\hline Appearance & Slightly pale yellow crystals \\
\hline Solubility & Soluble in DMSO, formation of yellow solution in methanol \\
\hline Density & $1.57 \pm 0.1 \mathrm{~g} / \mathrm{cm}^{3}$ (Predicted) \\
\hline pKa & $11.56 \pm 0.60$ (Predicted) \\
\hline Molar volume & 278.51 \\
\hline $\begin{array}{l}\text { Molecular polar surface } \\
\text { area }\end{array}$ & 155.14 \\
\hline Molecular 3D-polar SASA & 264.008 \\
\hline Molecular SASA & 523.67 \\
\hline Molar refraction & 82.12 \\
\hline
\end{tabular}

and terpenoids that can be generated through the MVA and MEP pathways. ${ }^{53}$ The same concept has been adopted for the biosynthesis of seco-iridoids. ${ }^{47,53}$ IPP and dimethylallyl diphosphate (DMAPP) condense together to form geranyl diphosphate (GPP), which is the starting precursor for the secoiridoid pathway for the biosynthesis of swertiamarin (Figures 4-6).

\section{Pharmacology and Therapeutic Potential of Swertiamarin}

Swertiamarin has been reported to possess many pharmacological activities. The underlying molecular mechanisms of action of the associated activities were identified through modulation of gene expression (Figures 7 and 8) and are summarized in Table 2.

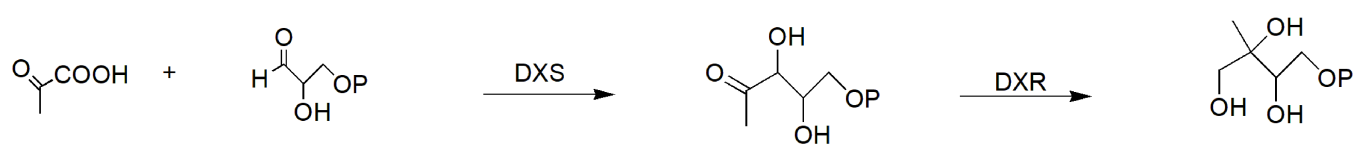

Pyruvate D-glyceraldehyde-3-phosphate 1-deoxy-D-xylulose-5-phosphate

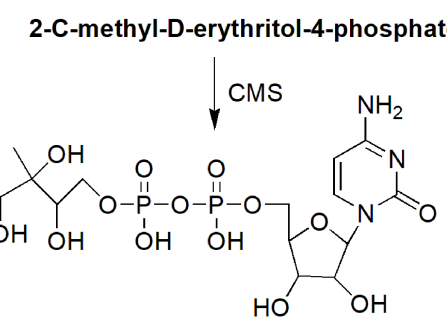

4-diphosphocytidyl-2-C-methyl-D-erythritol

4-diphosphocytidyl-2-C-methyl-D-erythritol-2-phosphate MECS

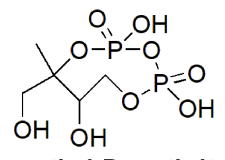

HDS

-C-methyl-D-erythritol-2,4-cyclodiphosphate

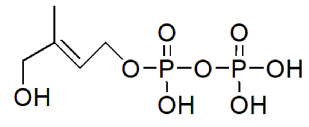

1-hydroxy-2-methyl-2-buteneyl-4-diphosphate

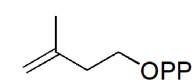

HMBPP reductase

Isopentenyl diphosphate (IPP)

Dimethylallyl diphosphate (DMAPP)

Figure 4 MEP pathway for the synthesis of isopentenyl diphosphate (IPP). 


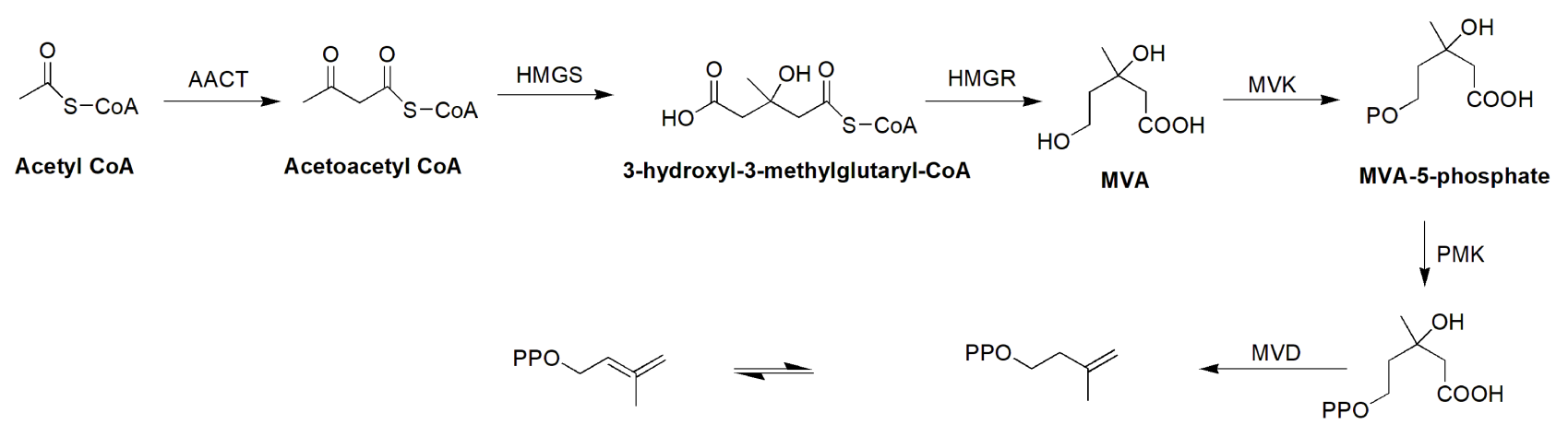

Dimethylallyl diphosphate (DMAPP)

Figure 5 MVA pathway for the synthesis of isopentenyl diphosphate (IPP).

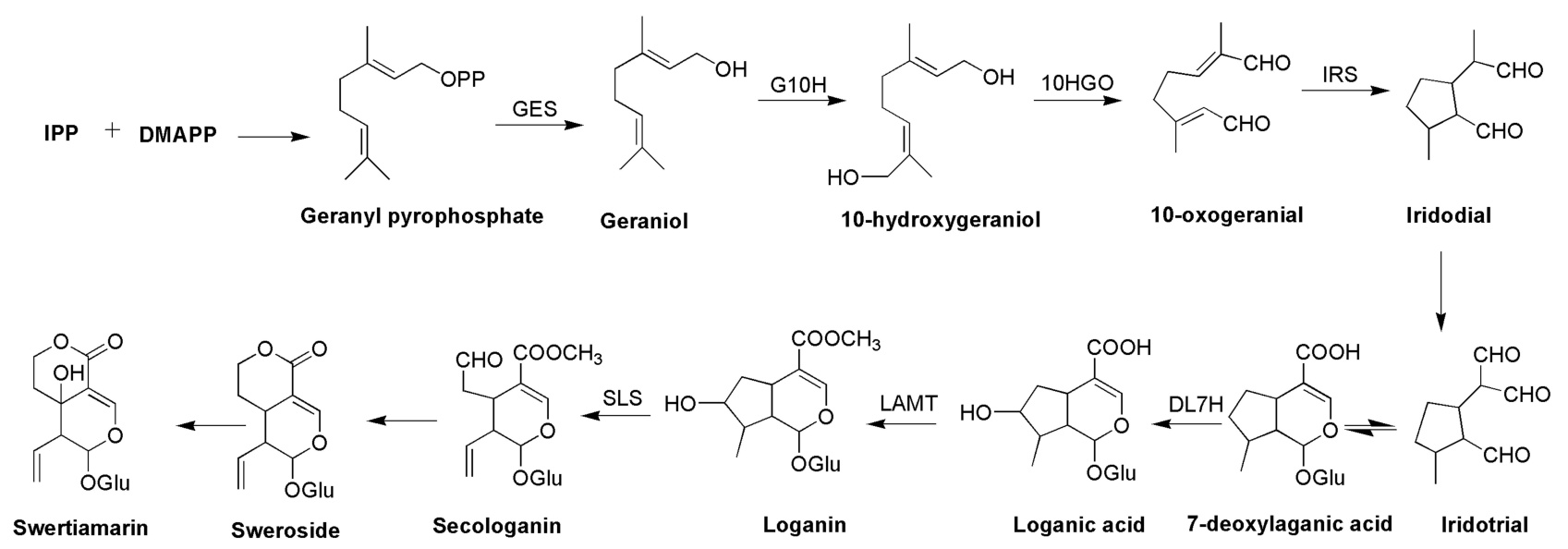

Figure 6 Biosynthetic pathway of swertiamarin (seco-iridoid pathway).

\section{Anti-Inflammatory and Antiarthritis Activities}

The protective effects of swertiamarin against inflammation-induced injury have been reported in several studies. Most recently, Zhang et $\mathrm{al}^{54}$ investigated the antiinflammatory mechanisms of swertiamarin using an azidelabeled swertiamarin probe. The probe was used for protein target fishing in which protein kinase B (AKT) was specifically captured. Immunofluorescence colocalization with AKT confirmed that AKT was targeted by swertiamarin. In addition, a set of AKT inhibitors was used, and a membrane translocation experiment was conducted to confirm the pleckstrin homology domain of AKT. This specific binding of swertiamarin directly deactivated the phosphorylation of AKT on both Ser473 and Thr308, which subsequently induced the dephosphorylation of IкB kinase enzyme (IKK) and nuclear factor kappa-light chain enhancer of activated B-cells (NF- $\kappa \mathrm{B})$. Swertiamarin treatment also suppressed the expression of proinflammatory cytokines, including tumor necrosis alpha (TNF- $\alpha$ ), interleukin (IL)-6 (IL-6), and IL-8, in cells (BEAS-2B cells, RAW264.7 cells and HEK 293T cells) and in an acute lung injury animal model by targeting the AKTpleckstrin homology (AKT-PH) domain. This study revealed that swertiamarin can act as a natural AKT inhibitor to regulate inflammatory conditions. ${ }^{54}$

In another study on arthritic rats by Saravanan et al, ${ }^{55}$ the effect of swertiamarin on the biochemical parameters, proinflammatory cytokines, enzymes and histopathology and radiography was investigated. The study confirmed that swertiamarin controlled the levels of free radicals, lysosomal enzymes, and bone destructive enzymes and modulated the paw thickness of arthritic rats. Additionally, swertiamarin treatment ameliorated bone loss and regulated inflammatory chemokines in the 


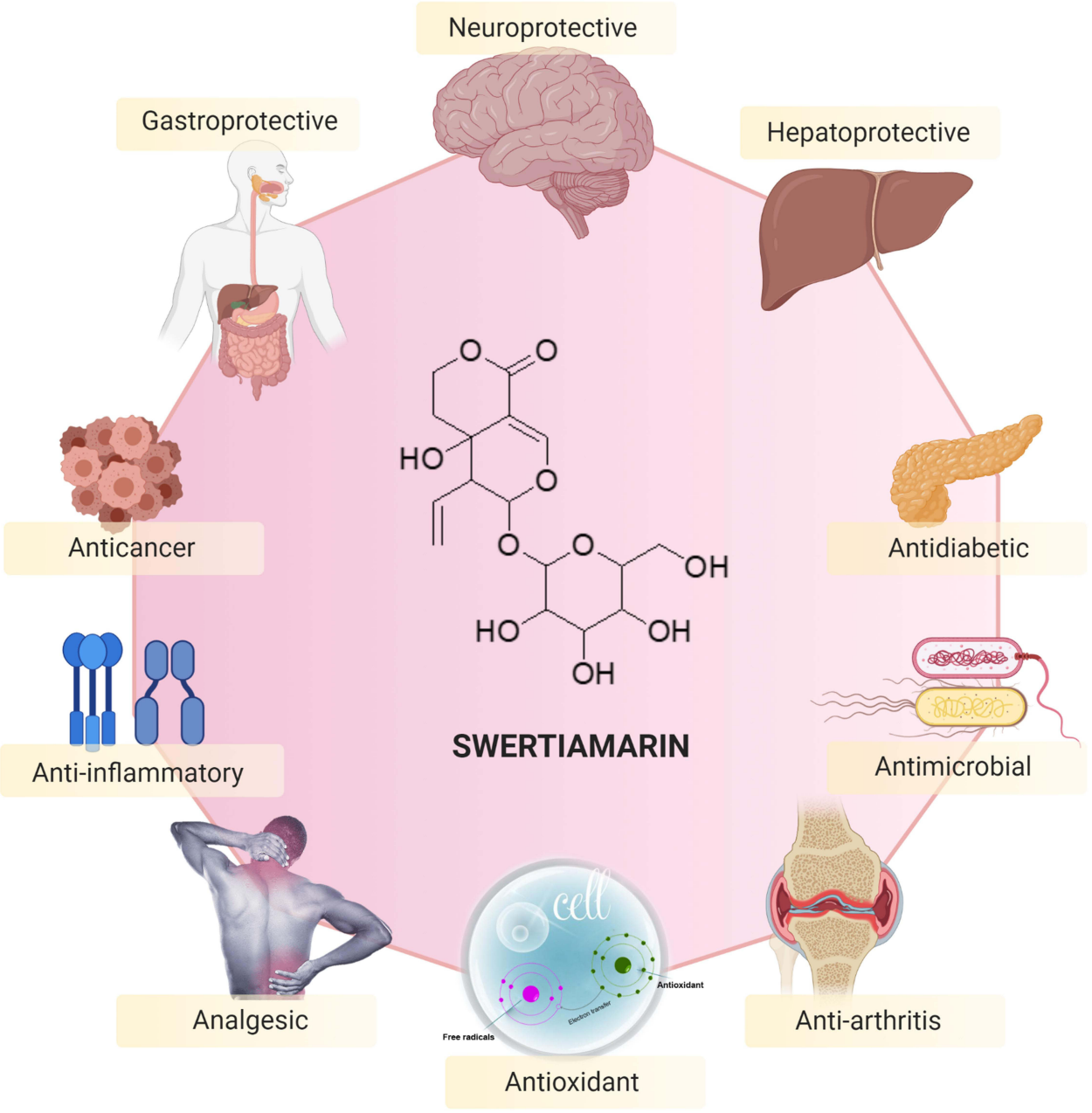

Figure 7 Pharmacological properties and therapeutic potential of swertiamarin.

Note: The figure was created with the support of https://biorender.com under the paid subscription.

affected joints, as evidenced by histopathological and radiological examinations. Additionally, the protein levels of p65, NF-kB, p-IkBa, p-STAT3 and p-JAK2 were markedly decreased after swertiamarin treatment, as shown in the animal models and lipopolysaccharide (LPS)-induced RAW 264.7 macrophage cells. The levels of the proinflammatory cytokines IL- $1 \beta$, TNF- $\alpha$ and IL- 6 were decreased, while IL-4 and IL-10 were increased in swertiamarin-administered arthritic rats compared to untreated animals. Furthermore, disease progression was also ameliorated by regulating the NF-kB/IkB and JAK2/STAT3 pathways, suggesting that swertiamarin is useful in the treatment of rheumatoid arthritis. ${ }^{55}$

Saravanan et $\mathrm{al}^{56}$ also investigated the antiinflammatory effect of swertiamarin in IL- $1 \beta$-induced fibroblast-like synoviocytes (FLSs) isolated from Freund's complete adjuvant-induced arthritic rats. The results showed that swertiamarin diminished IL-1 $\beta$ - induced cell proliferation $(149.46 \pm 13.73 \%)$ and nitric oxide production $(162.03 \pm 11.03 \%)$. Both the mRNA and protein levels of apoptotic markers (caspase-3), proinflammatory mediators [TNF- $\alpha$, IL-6, prostaglandin-E2 (PGE2), cyclooxygenase-2 (COX-2), nitric oxide synthase (iNOS), and matrix metallopeptidases (MMPs)] and osteoclastogenic mediators (RANKL) were expressed in a more regulated manner. In addition, swertiamarin treatment inhibited the levels of p38 MAPK in a dose-dependent manner but significantly reduced the levels in a time-dependent manner. Overall, the study confirmed the potential antiinflammatory activity of swertiamarin by ameliorating aggressive FLSs.

In addition, the immunomodulatory effect of swertiamarin was also studied by Saravanan et $\mathrm{al}^{57}$ using in vitro and in vivo models $(2,5$ and $10 \mathrm{mg} / \mathrm{kg} / \mathrm{day}$, P.O., for seven days). Higher HA titers, PFCs, and spleen and thymus weights were found in animals that received swertiamarin 


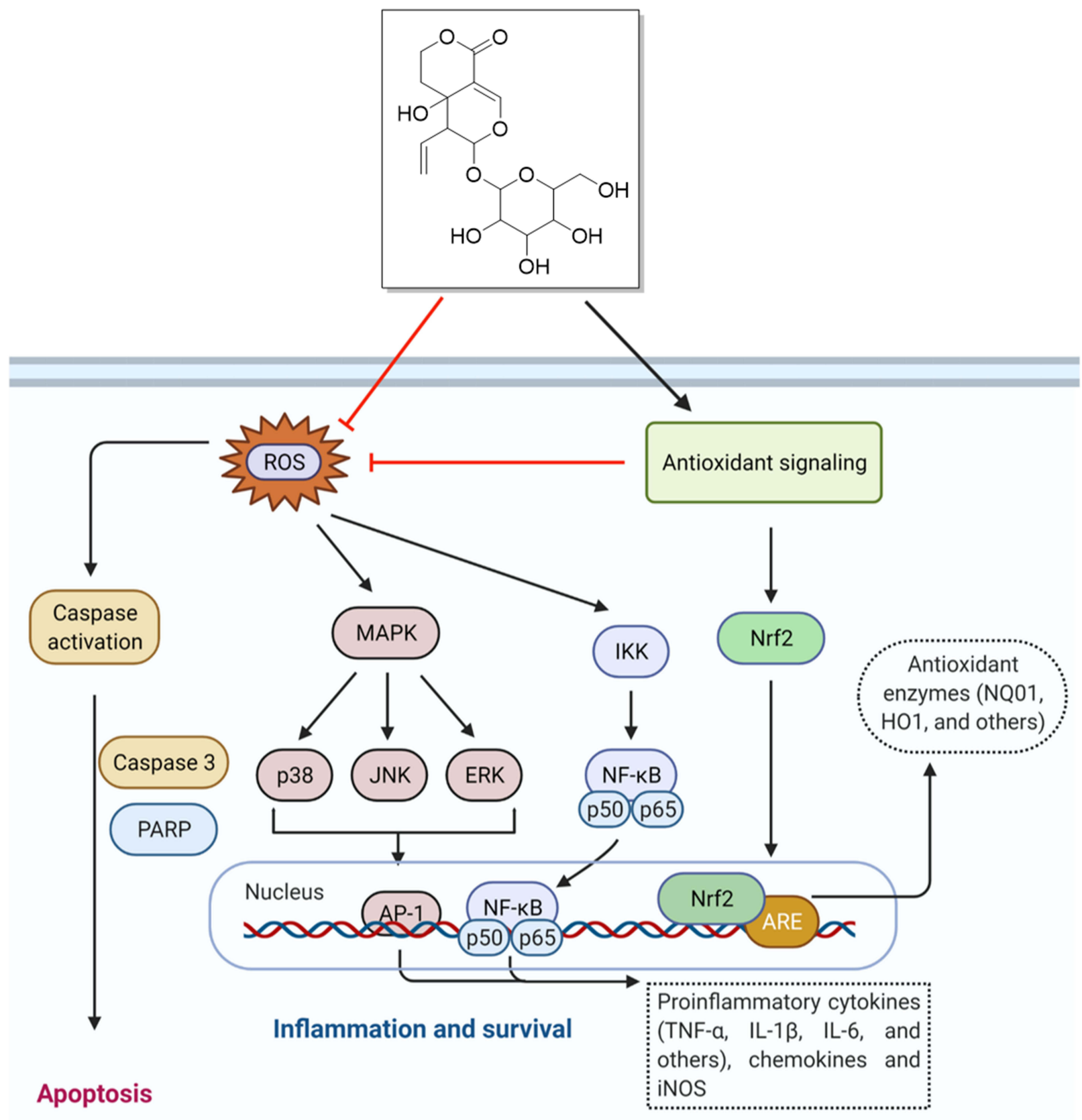

Figure 8 Molecular mechanism of action of swertiamarin.

Notes: Swertiamarin exhibits a broad spectrum of pharmacological activities that are mediated by diversified signaling pathways. However, antiapoptotic and antioxidant effects are the major contributors to swertiamarin. The role of other pathways has been deciphered in the main text. The figure was created with the support of https:// biorender.com under the paid subscription.

treatment, although fewer immunoinflammatory responses were seen in the delayed-type hypersensitivity test. The active compound swertiamarin also increased the mRNA and protein levels of Th2-mediated cytokines (IL-4 and IL-10) in animals. Additionally, swertiamarin administration diminished the levels of Th1-mediated cytokines
(TNF- $\alpha$, IL-1 $\beta$ and IL-6) in concanavalin A (Con A)stimulated splenocytes. Furthermore, phytohemagglutinininduced neutrophils could not generate free radicals, and the expression of proinflammatory cytokines (TNF-a, IL$1 \beta$ and IL-6) in LPS-induced macrophages was suppressed following swertiamarin administration. 
Table 2 An Overview of the Underlying Molecular Mechanisms for Different Pharmacological Properties of Swertiamarin

\begin{tabular}{|c|c|c|}
\hline $\begin{array}{l}\text { Pharmacological } \\
\text { Property }\end{array}$ & Mechanism & Reference \\
\hline $\begin{array}{l}\text { Anti-inflammatory and } \\
\text { antiarthritis }\end{array}$ & $\begin{array}{l}\text { - } \quad \downarrow \text { TNF- } \alpha \text {, IL-6 and IL-8 } \\
\text { - } \uparrow \text { IL-4 and IL-10 } \\
\text { - Inhibition of p38 and MAPK } \\
\text { - } \uparrow \text { mRNA of caspase-3 } \\
\text { - } \uparrow \text { Protein levels of Th2-mediated cytokines (IL-4 and IL-I0) } \\
\text { - } \downarrow \text { Proinflammatory cytokines (TNF- } \alpha, \text { IL-I } \beta \text { and IL-6) }\end{array}$ & $\begin{array}{l}\text { Zhang et al } ;{ }^{54} \text { Saravanan et al } ;{ }^{55} \\
\text { Saravanan et al } ;{ }^{56} \text { Saravanan et al }\end{array}$ \\
\hline Analgesic & - Not identified & Jaishree et al (2009) \\
\hline Hepatoprotective & $\begin{array}{l}\text { - Upregulation of Nrf2 } \\
\text { - } \downarrow \text { SREBP-I, FAS and acetyl-CoA carboxylase I } \\
\text { - Regulation of Bax, cleaved caspase-3 proteins, TGF- } \beta \text { I, collagen I, collagen } \\
\text { III, CTGF and fibronectin mRNA } \\
\text { - Regulation of Bcl-2 levels } \\
\text { - Regulation of hepatic PI3K/Akt pathway } \\
\text { - } \downarrow \text { MDA } \\
\text { - } \downarrow \text { Inflammatory cytokines (iNOS and IL-I } \beta \text { ) } \\
\text { - Upregulation of ATIR } \\
\text { - Inhibit Ang II-induced ERK }\end{array}$ & Yang et al; ${ }^{60}$ Zhang et al; ${ }^{61} \mathrm{Wu}$ et al ${ }^{62}$ \\
\hline Antidiabetic & $\begin{array}{l}\text { - } \uparrow \text { PPAR } \gamma / G L U T-4 \text { and adiponectin mRNA } \\
\text { - } \downarrow \text { Oxidative stress } \\
\text { - } \downarrow \text { Insulin resistance } \\
\text { - Upregulation of GLUT-2 } \\
\text { - } \downarrow \text { PEPCK } \\
\text { - } \alpha \text {-Glucosidase inhibitor }\end{array}$ & $\begin{array}{l}\text { Vaidya et al } ;{ }^{66} \text { Patel et al } ;{ }^{70} \text { Ahamad } \\
\text { et a }{ }^{72}\end{array}$ \\
\hline Antioxidant & $\begin{array}{l}\text { - } \downarrow \text { Cleavage of caspase-3 and PARPI } \\
\text { - } \downarrow \text { PEPCK; regulates AMPK } \\
\text { - } \downarrow \text { Oxidative stress } \\
\text { - } \downarrow \text { IR, PI3K, AKT in Ser (P)307 IRS-I }\end{array}$ & Patel et $\mathrm{al}^{73}$ \\
\hline Gastroprotective & - Inhibition of dopamine D2 receptor & Kimura and Sumiyoshi ${ }^{74}$ \\
\hline Lung protection & 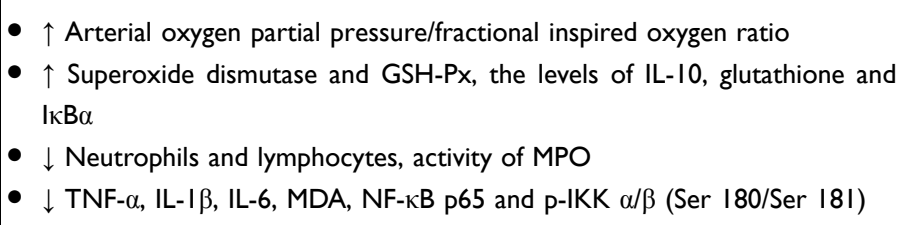 & Yao et $\mathrm{al}^{75}$ \\
\hline Neuroprotection & $\begin{array}{l}\text { - Upregulation of SOD-3 and GST-4 } \\
\text { - Downregulation of DAF-16 and SKN-I } \\
\text { - } \uparrow \text { IL-IO } \\
\text { - } \downarrow \text { TNF- } \alpha, \text { IL-6 and IL-I } \beta \\
\text { - Upregulation of DAF-I6 possibly via upregulation of unc-I7 }\end{array}$ & $\begin{array}{l}\text { Pandey et al; }{ }^{76} \text { Deng et al; } ; 7 \text { Wang } \\
\text { et a }\left.\right|^{84}\end{array}$ \\
\hline Anticancer & - Not identified & - \\
\hline
\end{tabular}

Note: The upward arrow $(\uparrow)$ indicates an increased response, and the downward arrow $(\downarrow)$ indicates a decreased response.

Swertiamarin treatment also has an antiosteoclastogenic role in preventing bone destruction by regulating inflammatory responses. Swertiamarin administration decreased the expression of tartrate-resistant acid phosphatase (TRAP), receptor activator of NF-kB ligand (RANKL) and receptor activator of NF-kB (RANK) levels and increased the levels of osteoprotegerin (OPG) in the in vivo model $(2,5$ and $10 \mathrm{mg} / \mathrm{kg}$, P.O. for 14 days), as well as in vitro $(50 \mu \mathrm{g} / \mathrm{mL})$ in 
osteoblasts and osteoclasts treated with Freund's complete adjuvant (FCA). In the osteoblast and osteoclast coculture system, swertiamarin treatment also modulated the levels of proinflammatory cytokines, MMPs and NF- $\mathrm{BB}$. In addition, in silico analysis showed that swertiamarin had an affinity for low binding energy residues of the proteins RANK, RANKL, and OPG. This research has revealed the antiosteoclastogenic role of swertiamarin in preventing bone destruction. $^{58}$ In an earlier study, Vaijanathappa and Badami $^{59}$ utilized rats for paw edema tests using carrageenan, formalin and histamine induction to investigate the antiedematogenic activity of swertiamarin (100 and $200 \mathrm{mg} / \mathrm{kg}$, P.O.). Interestingly, the anti-inflammatory effects of swertiamarin $(200 \mathrm{mg} / \mathrm{kg})$ tended to be superior to those of diclofenac sodium in all investigations purported to act via antioxidant mechanisms.

The mechanisms underlying the anti-inflammatory effects are primarily due to the inhibition of $N F-\kappa B$, which is a key inflammatory response mediator. In addition, the effect of swertiamarin on other tissue injury models should also be examined in future research. Therefore, further preclinical studies are needed in which sepsis and/or other models of tissue injury are treated with swertiamarin and its metabolites.

\section{Analgesic Activity}

The analgesic potential of swertiamarin has been reported by Jaishree et al. ${ }^{41}$ The study investigated the in vivo antinociceptive activity of swertiamarin (100 and $200 \mathrm{mg} / \mathrm{kg}$, P.O.) in mice and found that swertiamarin treatments significantly increased the latency period on a hot plate. The tail withdrawal reflex was increased in a dose-dependent manner, and a high percentage of pain protection was also observed. Interestingly, swertiamarin conferred better analgesic activity than standard paracetamol in both investigated methods. This finding suggests that swertiamarin may exert its antinociceptive activity by acting on both the peripheral and central nervous systems. Apart from this study, no other studies have reported the analgesic activity of swertiamarin. Thus, satisfactory mechanisms of action for the analgesic action of swertiamarin remain to be established.

\section{Hepatoprotective Activity}

Jaishree and Badami ${ }^{5}$ were the first to report the hepatoprotective effect of swertiamarin by evaluating its ability (100 and $200 \mathrm{mg} / \mathrm{kg} / \mathrm{day}$, P.O., for eight days) to protect rats with hepatic dysfunction caused by galactosamine
(d-GalN) (200 mg/kg, i.p., 8th day). Swertiamarin treatment reversed the alterations in biochemical parameters caused by d-GalN. Interestingly, swertiamarin also protected against nonalcoholic fatty liver disease (NAFLD) in fructose-fed mice, as reported by Yang et al. ${ }^{60}$ Following the administration of swertiamarin $(25,50$ and $100 \mathrm{mg} / \mathrm{kg} / \mathrm{day}$, intragastric, for four weeks), the plasma levels of uric acid, alanine aminotransferase (ALT), triglycerides, serum glucose, and aspartate aminotransferase (AST) were decreased. Based on the histological evaluations, swertiamarin also relieved hepatic ballooning degeneration. Oxidative stress and the levels of proinflammatory cytokines were reduced in the liver, causing a slowing down of xanthine oxidase (XO) enzymatic action and strengthening the defensive mechanism together with activation of nuclear factor E2-related factor 2 (Nrf2) in fructose-administered mice. Moreover, swertiamarin treatment also suppressed the expression of fatty acid synthase (FAS), sterol-regulatory element-binding protein-1 (SREBP-1), and acetyl-CoA carboxylase 1 in the liver of fructose-fed mice. Thus, it was concluded that swertiamarin can improve NAFLD by regulating carbohydrate metabolism.

In another study, swertiamarin was administered to Sprague-Dawley rats with hepatic impairment induced by carbon tetrachloride $\left(\mathrm{CCl}_{4}\right)$ to study its possible pathways. ${ }^{61}$ The rats were administered $40 \% \mathrm{CCl}_{4}$ via a subcutaneous injection followed by swertiamarin (100 and $200 \mathrm{mg} / \mathrm{kg} /$ day, P.O., for eight weeks). There was a remarkable decrease in the number of TUNEL-positive cells in swertiamarin-treated animals compared to untreated rats. Both the mRNA and protein levels of Bax, cleaved caspase-3 proteins, transforming growth factor beta-1 (TGF- $\beta 1$ ), collagen I, collagen III, connective tissue growth factor (CTGF) and fibronectin were decreased after swertiamarin treatment. Furthermore, the increased levels of Bcl-2 stimulated by swertiamarin reversed the effect of carbon $\mathrm{CCl}_{4}$. Additionally, the phosphatidylinositol-3-kinase/protein kinase $\mathrm{B}$ (PI3K/Akt) pathway, responsible for hepatic cell survival, was also suppressed, suggesting that swertiamarin has the potency to alleviate murine hepatic cell death.

Another study also confirmed the hepatoprotective effect of swertiamarin on $\mathrm{CCl}_{4}$-induced liver failure. ${ }^{62}$ Swertiamarin administration (100 and $200 \mathrm{mg} / \mathrm{kg} / \mathrm{day}$, P.O., for eight weeks) alleviated serum ALT, AST, and alkaline phosphatase (ALP) levels and ameliorated liver cellular changes caused by $\mathrm{CCl}_{4}$. Free radical-stimulated 
damage in the liver was also decreased, as evidenced by decreased malondialdehyde (MDA) levels along with increased levels of antioxidants, including superoxide dismutase, glutathione peroxidase, and glutathione. In addition, swertiamarin ameliorated the liver injury caused by $\mathrm{CCl}_{4}$-induced inflammation by reducing the levels of inducible iNOS and IL-1 $\beta$. Cytochrome P450 (CYP), efflux transporters and the PDZK1 gene were more significantly expressed in the swertiamarin-treated group. Therefore, it was concluded that swertiamarin protects rat livers from $\mathrm{CCl}_{4}$-induced inflammation by acting as an antioxidant and activator of detoxification enzymes and promoting the expression of efflux transporters via the nuclear Nrf2/ heme oxygenase-1 (HO-1) pathway. ${ }^{62}$

It has been reported that the development of hepatic fibrosis depends on the renin-angiotensin system (RAS), making it the focus in developing a favorable therapy. $\mathrm{Li}$ et $\mathrm{al}^{63}$ investigated the effects of swertiamarin (15 and $20 \mathrm{mg} / \mathrm{kg} /$ day, i.p., for two weeks) on dimethylnitrosamine (DMN) $(10 \mu \mathrm{g} / \mathrm{kg}$, i.p., 3 days/week for four weeks) and confirmed that its primary mechanisms were by acting on RAS. Swertiamarin significantly inactivated hepatic stellate cell (HSC) growth due to angiotensin II (Ang II). Additionally, swertiamarin repressed DMN-induced $\alpha$ smooth muscle actin synthesis and improved liver function. Ang II-induced angiotensin type 1 receptor (AT1R) and extracellular signal-regulated kinase (ERK), as well as c-jun N-terminal kinase (JNK) phosphorylation in HSCs, were inhibited by swertiamarin. Swertiamarin ameliorated the inhibition of plasma Ang II in rats exposed to DMN. A similar effect was also shown in murine liver in terms of DMN-induced AT1R upregulation and ERK and JNK phosphorylation. Therefore, it was concluded that swertiamarin may attenuate hepatic fibrosis by inhibiting HSC activation via RAS regulation.

Several animal experiments to detect the protective effects of swertiamarin against hepatotoxic agents have been summarized in this review. Swertiamarin serves as an antidote to hepatotoxicity caused by chemical compounds, according to the findings of various studies. In particular, swertiamarin demonstrated anti-inflammatory activity in the liver by decreasing TGF- $\beta 1$ protein expression. In addition, multiple pathways, such as oxidative stress inhibition, inflammation, and apoptosis, are involved in the hepatoprotective effects of swertiamarin. However, there is a need for more research to examine the hepatoprotective effects of swertiamarin on human intoxication and from the perspective of mitochondrial damage that should be discussed in the future.

\section{Antidiabetic Activity}

Some medicinal plants, including Gentiana kurroo, ${ }^{18}$ E. littorale, $^{40}$ Swertia longifolia, ${ }^{32}$ and Swertia chirayita, ${ }^{64}$ which contain swertiamarin as one of the major ingredients, have been reported to have antidiabetic properties. Numerous in vivo studies have been performed to investigate the antidiabetic properties of swertiamarin and the underlying mechanism of action.

Ghazanfar et $\mathrm{al}^{18}$ investigated the antidiabetic effect of methanolic and hydroethanolic extracts of Gentiana kurroo Royle. The results showed that decreased serum glucose concentrations in streptozotocin (STZ)-induced diabetic rats were detected, which may be attributed to the presence of swertiamarin, which has been reported as one of the major constituents of Gentiana kurroo Royle. ${ }^{65}$ Additionally, the levels of other related biochemical parameters, such as triglycerides, ALP, creatinine, cholesterol, low-density lipoproteins (LDL), pyruvate transaminases, and serum glutamate oxaloacetate, were also ameliorated. Interestingly, regeneration of $\beta$-cells in the pancreas of diabetic rats confirmed the potential antidiabetic effect of the extract, which is probably contributed by swertiamarin.

In addition, Saeidnia et $\mathrm{al}^{32}$ investigated the $\alpha$ amylase inhibitory activity of some genera containing swertiamarin and proposed that they possess good antidiabetic candidates that have the potential to be formulated. Patel et $\mathrm{al}^{40}$ reported the antihyperglycemic and antihyperlipidemic properties of aqueous and ethanolic extracts of E. littorale. Swertiamarin can metabolize into an active compound called gentianine that has a pharmacophoric moiety (Figure 9). Vaidya et $\mathrm{al}^{66}$ investigated whether gentianine could contribute to the antidiabetic effect of swertiamarin. There was no major impact of swertiamarin on adipogenesis or the mRNA expression of peroxisome proliferator-activated receptors gamma $(P P A R-\gamma)$ and glucose transporter-4 (GLUT-4); however, there was a substantial increase in adiponectin mRNA expression. Comparatively, gentianine treatment substantially improved adipogenesis, which was associated with a substantial increase in the mRNA levels of PPAR- $\gamma$, GLUT-4 and adiponectin, suggesting that the antidiabetic function of swertiamarin is contributed by its active metabolite gentianine.

The benefits of swertiamarin on dyslipidemic conditions associated with type 2 diabetes in streptozotocininduced $(90 \mathrm{mg} / \mathrm{kg}$, i.p.) rats have been explored by Vaidya et al. ${ }^{67}$ Swertiamarin administration $(50 \mathrm{mg} / \mathrm{kg} /$ 


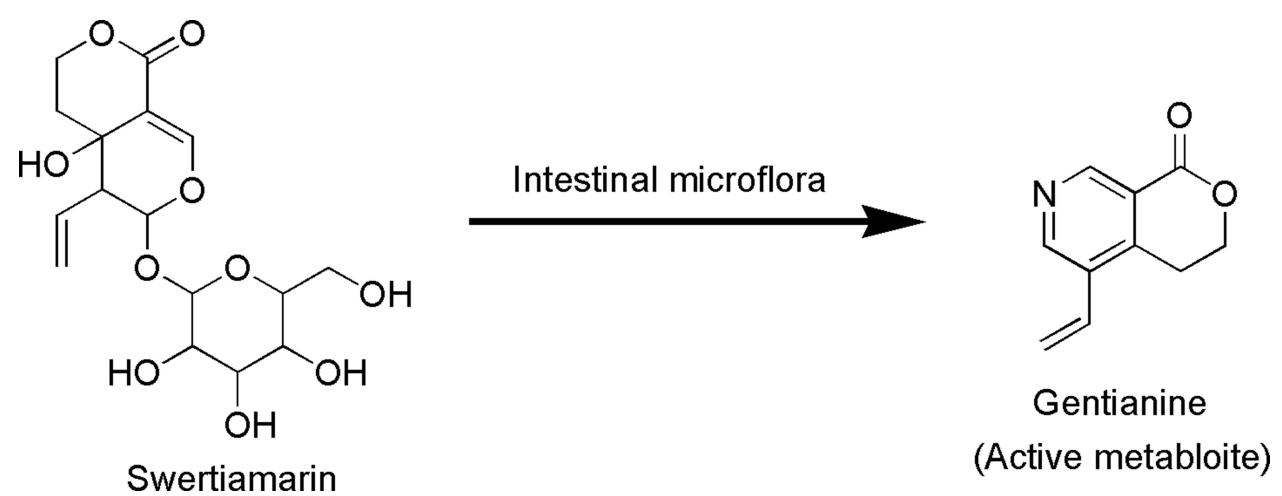

Figure 9 Metabolic conversion of swertiamarin to gentianine by intestinal microflora.

day, i.p., for six weeks) markedly decreased serum triglycerides, cholesterol and LDL levels. Similarly, there was a notable decline in fasting glucose levels. Furthermore, the elevation of the insulin sensitivity index was significantly observed in rats that received swertiamarin treatment. Taken together, the findings suggest that swertiamarin exerts beneficial effects on diabetic complications such as dyslipidemia. Vaidya et $\mathrm{al}^{68}$ also reported that swertiamarin could alleviate diabetes and its cardiovascular complications, such as atherosclerosis and renal failure, in Zucker fa/fa rats. The levels of serum glucose, triglycerides, nonesterified free fatty acids and cholesterol were decreased compared to those in untreated rats upon swertiamarin treatment $(75 \mathrm{mg} / \mathrm{kg} / \mathrm{day}$, i.p., for 28 days). A similar effect was also seen in the reduction of serum MMP-9, MMP-3 and urea levels.

In addition, Dhanavathy ${ }^{69}$ also investigated the antidiabetic activity of swertiamarin in STZ-induced $(50 \mathrm{mg} / \mathrm{kg}$, i.p.) diabetic rats. Swertiamarin treatment $(15,25$ and $50 \mathrm{mg} / \mathrm{kg} /$ day, P.O., 28 days) resulted in a remarkable reduction in fasting blood glucose, $\mathrm{HbAlc}$, total cholesterol, total glycerides, and LDL levels. The levels of hemoglobin, plasma insulin, total protein, body weight and HDL were also significantly increased compared to STZ-induced diabetic rats, indicating that swertiamarin can ameliorate hyperglycemia and its complications.

The therapeutic effect of swertiamarin in non-insulindependent diabetes mellitus rats was investigated by Patel et al. ${ }^{70}$ Swertiamarin $(50 \mathrm{mg} / \mathrm{kg} /$ day, P.O., for 40 days $)$ and aqueous extract of $E$. littorale ( $15 \mathrm{~g}$ of dried plant equivalent extract $/ \mathrm{kg}$, P.O., for 40 days) restored the activity of glucose-6-phosphatase and 3-hydroxy-3-methyl-glutarylcoenzyme A reductase (HMG-CoA reductase) while maintaining the physiological transcriptional levels of phosphoenolpyruvate carboxykinase (PEPCK), glucokinase (GCK), glucose transporter type 2 (GLUT-2), PPAR$\gamma$, leptin $(L E P)$, adiponectin $(A D I P O Q)$, lipoprotein lipase $(L P L)$, sterol regulatory element-binding transcription factor-1c (SREBP-1c) and glucose transporter type 4 (GLUT4) genes. These findings suggest that both treatments improved insulin sensitivity and controlled carbohydrate and fat metabolism.

Furthermore, the therapeutic effect of $E$. littorale aqueous extract $(1 \mathrm{~g} / \mathrm{kg} / \mathrm{day}$, P.O., for three weeks) and swertiamarin $(50 \mathrm{mg} / \mathrm{kg} / \mathrm{day}$, P.O., for three weeks) on rats with type 1 diabetic nephropathy (DN) complications has also been investigated. ${ }^{71}$ Serum urea and creatinine and other parameters linked to the progression of DN type 1 were markedly reduced after the treatments. Glomerular function was also substantially enhanced at the cellular level in rats treated with aqueous extracts of E. littorale and swertiamarin.

Finally, Ahamad et al ${ }^{72}$ used in vitro and in vivo models to determine the potential of swertiamarin in maintaining glucose homeostasis via the inhibition of carbohydrate (starch and sucrose)-metabolizing enzymes. The results showed that swertiamarin (20 mg/kg, P.O.) could inhibit $\alpha$ amylase and $\alpha$-glucosidase with minimum $50 \%$ inhibitory concentration $\left(\mathrm{IC}_{50}\right)$ values of $1.29 \pm 0.25 \mathrm{mg} / \mathrm{mL}$ (starchfed mice) and $0.84 \pm 0.11 \mathrm{mg} / \mathrm{mL}$ (sucrose-fed mice), respectively. Studies in carbohydrate-challenged mice indicated that swertiamarin effectively ameliorated the increase in peak blood glucose levels. The increase in peak blood glucose levels was only $49 \mathrm{mg} / \mathrm{dl}$ (starch-fed mice) and $57 \mathrm{mg} / \mathrm{dl}$ (sucrose-fed mice) in the swertiamarin-treated groups compared to $70 \mathrm{mg} / \mathrm{dl}$ (starch-fed mice) and $80 \mathrm{mg} / \mathrm{dl}$ (sucrose-fed mice) in the untreated groups. 
While all the available in vitro and in vivo studies indicate that swertiamarin has good potential to be used in the treatment of diabetes, we understand the need for clinical studies. It is important to further investigate studies focusing on the effective dose of swertiamarin and the comprehensive effects on insulin, lipid, plasma glucose and $\mathrm{HbA} 1 \mathrm{c}$ levels. Further analysis of the mechanism of swertiamarin in diabetes should pay attention to the relationship between different mechanisms.

\section{Antioxidant Activity}

Several preclinical studies have indicated that swertiamarin possesses good antioxidant properties. To date, most experimental models reported in the literature have investigated the use of swertiamarin in animal models with preexisting pathologies. Since the main therapeutic effect of swertiamarin is glucose regulation and liver protection, as described in the previous sections of this review, most studies have utilized diabetic animal and hepatotoxicityinduced models to address the protective role of swertiamarin as an antioxidant in affected organs, such as the pancreas, liver or kidneys.

In an earlier study, Vaijanathappa and Badami ${ }^{59}$ utilized seven different in vitro methods to evaluate the antioxidant properties of swertiamarin. They observed potent antioxidant activity based on 2,2'-azino-bis(3-ethylbenzothiazoline-6-sulfonic acid) (ABTS) and hydrogen peroxide methods. Moderate activity in deoxyribose and lipid peroxidation assays with low $\mathrm{IC}_{50}$ values was observed in which the total antioxidant capacity of swertiamarin was $4.51 \mathrm{mM}$ ascorbic acid per gram, indicating that swertiamarin is a good antioxidant.

Patel et $\mathrm{al}^{73}$ demonstrated the antioxidant and lipidlowering properties of swertiamarin. Swertiamarin also improved NAFLD-related conditions, including the accumulation of fat in the liver, inflammation and impaired insulin sensitivity. Briefly, oleic acid (1 mM) was administered for 24 hours to mediate steatosis in HepG2 cells, as confirmed by the significant accumulation of lipid droplets in Oil Red O (ORO) staining and triglyceride accumulation. Administration of swertiamarin $(25 \mu \mathrm{g} / \mathrm{mL})$ decreased triglyceride levels, membrane integrity and LDH secretion to prevent cell death due to reduced cleavage of caspase- 3 and poly (ADPribose) (PARP1). In addition, swertiamarin increased the expression of major insulin signaling proteins, such as insulin receptor (IR), PI3K, and pAkt, with a concomitant reduction in p307 IRS-1. Swertiamarin also activated adenosine monophosphate-activated protein kinase (AMPK) phosphorylation, which led to restoration of insulin sensitivity in HepG2 cells. Furthermore, oleic acid upregulated sterol regulatory element binding protein (SREBP)-1c and fatty acid synthase $(F A S)$, resulting in increased synthesis of fatty acids. PPAR- $\alpha$, a major potential modulator of carbohydrate metabolism, is effectively regulated by swertiamarin to reduce the levels of the gluconeogenic enzyme PEPCK, limiting gluconeogenesis and fatty acid synthesis in the liver. Collectively, swertiamarin targets the potential metabolic regulator AMPK to promote a more controlled hepatic glycemic burden, fat accumulation, insulin resistance and amelioration of reactive oxygen species (ROS) in hepatic steatosis. These findings indicate the potential use of swertiamarin in regulating metabolism clinically and as a promising novel therapeutic agent in the treatment of NAFLD.

The biological characteristics of swertiamarin with a typical antioxidant action mechanism are highlighted in this review. The above results indicate that swertiamarin has been successful in improving antioxidant levels under oxidative stress conditions.

\section{Gastroprotective Activity}

There is only one study investigating the gastroprotective effects of swertiamarin ( 25 and $50 \mathrm{mg} / \mathrm{kg}$, P.O.) on gastric emptying and gastrointestinal motility ${ }^{74}$ in mice administered atropine, dopamine and 5-hydroxytryptamine (5-HT). The results indicated that gastric emptying and gastrointestinal motility stimulated by dopamine $(1 \mathrm{mg} /$ $\mathrm{kg}$, i.p.) were inhibited by swertiamarin at a dose of $50 \mathrm{mg} / \mathrm{kg}$. However, swertiamarin stimulated gastric emptying and gastrointestinal motility induced by $5-\mathrm{HT}(4 \mathrm{mg} /$ $\mathrm{kg}$, i.p.). It is plausible that swertiamarin acts as a dopamine D2 receptor inhibitor while not affecting the 5-HT receptor, therefore indicating its potential effect on functional dyspepsia. Nevertheless, the mechanism of action underlying gastric emptying and gastrointestinal motility remains to be elucidated. Aside from this review, no other studies have been published to the best of our knowledge on the protective effects of swertiamarin against esophageal and gastric disorders. It is therefore proposed that swertiamarin be used in the study of a wide range of gastrointestinal tract properties, such as reflux esophagitis defense, Barrett's esophagus, and damage to the gastric mucosa caused by certain antiinflammatory drugs and necrotizing agents. 


\section{Protection Against Lung Injury}

The protective effect and possible mechanisms of swertiamarin against a sepsis-induced acute lung injury model in rats were evaluated. ${ }^{75}$ The results showed that swertiamarin significantly increased the arterial oxygen partial pressure/fractional inspired oxygen ratio, the activities of superoxide dismutase and GSH-Px and the levels of IL-10, glutathione and I $\kappa \mathrm{B} \alpha$ followed by decreasing the lung wet to dry ratio, the neutrophil and lymphocyte counts, the protein content, the activity of MPO and the levels of TNF- $\alpha$, IL-1 $\beta$, IL-6, MDA, NF- $\kappa B, p 65$ and p-IKK $\alpha / \beta$ (Ser 180/Ser 181). The survival rate of rats with cecal ligation and puncture (CLP)-induced acute lung injury was significantly increased following treatment with swertiamarin. Swertiamarin also showed a protective effect against CLP-induced acute lung injury in rats by reducing blood capillary membrane permeability, inhibiting the inflammatory response and increasing antioxidant ability. No other studies have been published to support the notion, apart from this research, that swertiamarin plays a protective role in lung injury or disorders. It is recommended that swertiamarin also be tested for its effects on chronic obstructive pulmonary disease (COPD), acute respiratory distress syndrome (ARDS) and allergic asthma in the future, with the therapeutic effect of preventing or modulating inflammation and oxidative stress.

\section{Neuroprotective Activity}

The protective role of swertiamarin in enhancing cholinergic dysfunction has been elucidated by Pandey et al. ${ }^{76}$ Using Caenorhabditis elegans as a model, swertiamarin enhanced neurotransmission by modulating the function of acetylcholinesterase and nicotinic acetylcholine receptors (nAChRs) via unc-17 and unc-50 upregulation. It was shown that swertiamarin inhibited $\mathrm{AChE}$, as evidenced in both in vivo and cell-free systems. The in silico molecular docking of swertiamarin and human AChE exhibited strong binding energy $(-6.02)$. Interestingly, the increase in aldicarb and levamisole sensitivity post swertiamarin treatment was curtailed to a significant level in $D A F-16$ and $S K N-1$ mutants. Additionally, swertiamarin increased the levels of endogenous antioxidant enzymes by upregulating SOD-3 and GST-4 to provide protection against neurodegeneration, indicating that swertiamarin can boost cholinergic dysfunction. In addition, the study also indicated that swertiamarin might elicit an antioxidant response through the upregulation of $D A F-16$, possibly via upregulation of unc-17.
In addition, the anticonvulsant activity of swertiamarin $\left(50,150\right.$ and $450 \mathrm{mg} / \mathrm{kg}$, i.p.) was reported by Deng et al ${ }^{77}$ in mice treated with pilocarpine $(280 \mathrm{mg} / \mathrm{kg}$, i.p. $)$. Swertiamarin pretreatment (150 and $450 \mathrm{mg} / \mathrm{kg}$. i.p.) delayed the onset of the first convulsion and decreased the incidence of status epilepticus and mortality. There was a significant decrease in epileptic discharges based on electroencephalogram (EEG) analysis, where the damage in neurons was alleviated by similar doses of swertiamarin, as observed by Nissl and Fluoro-Jade $\mathrm{B}$ staining. Activated astrocytes can release a large number of proinflammatory cytokines, including IL-1 $\beta$, IL-6, and TNF- $\alpha$, as an important source of immunologically relevant cytokines that are highly related to epilepsy development. ${ }^{78,79}$ These proinflammatory cytokines are expressed in the normal brain at low levels and are extensively upregulated in patients with epilepsy. ${ }^{80}$ In addition, these cytokines alter neuronal excitability and reduce the seizure threshold, further establishing hyperexcitability of the chronic neuronal network and generating recurrent spontaneous seizures. ${ }^{81}$ Additionally, the administration of IL-10 can effectively prevent the development of epilepsy. ${ }^{82}$ The highest concentration of swertiamarin $(450 \mathrm{mg} / \mathrm{kg}$, i.p.) markedly abolished the activation of astrocytes. Additionally, Western blot analysis confirmed reduced expression of IL-1 $\beta$, IL-6, and TNF- $\alpha$ and increased expression of IL-10, indicating that swertiamarin exhibits anticonvulsant effects on pilocarpine-treated mice.

Similarly, Vaijanathappa et al ${ }^{83}$ explored the anticonvulsant activity of swertiamarin (10, 20 and $30 \mathrm{mg} / \mathrm{kg}$, i.p.) in a pentylenetetrazole-induced $(80 \mathrm{mg} / \mathrm{kg}$, i.p.) mouse model. At a low dose $(10 \mathrm{mg} / \mathrm{kg})$, swertiamarin provided defense against convulsion at $33.3 \%$ in a dose-dependent manner, with $66.00 \%$ and $72.67 \%$ protection against tonicclonic convulsions at 20 and $30 \mathrm{mg} / \mathrm{kg}$, respectively. In addition, the higher dose of swertiamarin $(30 \mathrm{mg} / \mathrm{kg})$ yielded a $27.33 \%$ mortality rate against pentylenetetrazoleinduced convulsion in albino mice. Therefore, this finding suggests that a lower dose of swertiamarin is preferable for its neuroprotective activity.

The potential of swertiamarin in preventing ischemia/ reperfusion injury was also investigated using mouse middle cerebral artery occlusion (MCAO) and primary hippocampal neuron oxygen-glucose deprivation/reperfusion (OGD/R) models. ${ }^{84}$ Before establishing the MCAO model, the mice were pretreated with swertiamarin (25, 100 and $400 \mathrm{mg} / \mathrm{kg} / \mathrm{day}$, i.p., for seven days), while hippocampal neurons were maintained in swertiamarin $(0.1,1$ 
or $10 \mu \mathrm{M})$ during reoxygenation. A notable reduction in infarct volume, dead nerve cells and free radical damage, as well as improvement in the recovery of neurological function in vivo, was observed after swertiamarin administration. Swertiamarin also minimized the amount of ROS and enhanced cell survival. Pretreatment with swertiamarin also improved Nrf2 nuclear translocation and upregulated the expression of $\mathrm{NAD}(\mathrm{P}) \mathrm{H}$ : quinone oxidoreductase-1 (NQO1) hemeoxygenase-1 (HO-1) both in vivo and in vitro. These effects were reversed by an Nrf2 inhibitor. Covalent molecular docking suggested the binding of swertiamarin to Keap1. In summary, swertiamarin activates the Nrf2 protective pathway to repress oxidative damage, thus making it a potential therapeutic agent against cerebral ischemia/reperfusion injury. The neuroprotective effects of swertiamarin have been reported in limited studies, and the mechanism is still unclear. Thus, more detailed investigations related to its neuroprotection and its mechanisms are required in the future.

\section{Anticancer Activity}

Swertiamarin has also been reported to have anticancer activity. For instance, Tang et $\mathrm{al}^{85}$ exposed hepatocellular carcinoma (HCC) cells to swertiamarin and conducted gene expression profiling. Cell survival and migration were minimized in swertiamarin-treated mice, while HepG2 cell death was induced. There was significant suppression of SK-Hep-1 cell growth xenografts in nude mice. It was plausible that the most crucial modulated pathway was PI3K-AKT, as evidenced in HepG2 cells. Estimation of 47 targets of swertiamarin was conducted by chemical genomics-based virtual screening, while the three-nearest neighbors method was used to estimate 21 targets. Both programs predicted eight targets for swertiamarin, and two of them (JUN and STAT3) that could modulate a broad range of differentially expressed genes (DEGs) are important. The study concluded that swertiamarin treatment could regulate the expression of several genes that are involved in cell viability, the cell cycle, apoptosis and invasion. Moreover, the majority of these investigated genes can be clustered into pathway networks, including PI3K, JUN, and STAT3, which are expected targets of swertiamarin. Nevertheless, the exact mechanism of swertiamarin in combating tumors should be confirmed to aid in the development of a novel formulation for cancer treatment.
In a recent study, Vaijanathappa et $\mathrm{al}^{83}$ investigated the cytotoxicity of swertiamarin on HEp-2 and HT-29 cells via in vitro MTT and sulfate-reducing bacteria (SRB) assays. The $\mathrm{IC}_{50}$ values of swertiamarin in HepG2 cells were 35.00 $\pm 0.68 \mu \mathrm{g} / \mathrm{mL}$ (based on the MTT assay) and 56.01 \pm 0.75 $\mu \mathrm{g} / \mathrm{mL}$ (SRB). In contrast, the $\mathrm{IC}_{50}$ values in HT-29 cells were $50.00 \pm 1.00(\mathrm{MTT})$ and $100 \pm 00 \mu \mathrm{g} / \mathrm{mL}(\mathrm{SRB})$.

In addition, the anticancer potential of swertiamarin in SK neuroblastoma cells has also been evaluated. ${ }^{86}$ The MTT results revealed that the $\mathrm{IC}_{50}$ value of swertiamarin was $19 \pm 2.27 \mathrm{mM}$. The $10 \mu \mathrm{M}$ human serum albumin (HSA) $/ \alpha-1$ acid glycoprotein (AGP) ligand concentrationinduced apoptosis in NK neuroblastoma cells was half of the $\mathrm{IC}_{50}$ value of swertiamarin. Additionally, different biophysical studies and in silico studies were conducted to validate the interaction between human plasma proteins and swertiamarin in which swertiamarin was highly bound to HSA and AGP. It was also found that swertiamarin binding led to protein conformational changes due to partial unfolding of the protein, indicating the anticancer effect of swertiamarin. ${ }^{86}$

The study of the anticancer effects of natural products is an active area of research. Although it has been proven that swertiamarin has anticancer activity, most of the studies are based on in vitro experiments, but to the best of our knowledge, no in vivo or clinical research has been conducted. In the future, attention needs to be paid to the combination of in vitro and in vivo anticancer studies.

\section{Antimicrobial Activity}

The antimicrobial activity of swertiamarin has been investigated by Šiler et al. ${ }^{87}$ Their team investigated its antimicrobial activity against eight bacterial species, consisting of four gram-negative bacteria, namely, Pseudomonas aeruginosa, Escherichia coli, Enterobacter cloacae and Salmonella typhimurium, and four grampositive bacteria, namely, Bacillus cereus, Listeria monocytogenes, Staphylococcus aureus and Micrococcus flavus, together with five fungal species (Aspergillus niger, Aspergillus versicolor, Trichoderma viride, Penicillium funiculosum and Aspergillus fumigatus). Based on this study, swertiamarin was confirmed to have strong antibacterial activity with a minimum inhibitory concentration (MIC) of $0.01-0.04 \mathrm{mg} / \mathrm{mL}$ and antifungal activity with an $\mathrm{MIC}$ of $0.001-0.1 \mathrm{mg} / \mathrm{mL}$ against all investigated organisms.

Few studies on the antimicrobial activity of swertiamarin have been performed, while encouraging findings 
against certain bacteria and fungi have been shown. This review indicated that more antimicrobial studies of swertiamarin could be useful in the future for the production of a novel and natural antimicrobial agent.

\section{Toxicity Profile of Swertiamarin}

The toxicity profile of swertiamarin has also been reported recently by Dhanavathy and Jayakumar ${ }^{88}$ by using acute and subchronic toxicity studies. In the acute toxicity study, the rats were treated with different oral doses of swertiamarin $(5-2000 \mathrm{mg} / \mathrm{kg})$. The results showed that swertiamarin did not produce any significant toxicity, behavioral responses, physiological changes, physical observations (skin, fur, eye mucous membrane, behavior patterns, tremors, salivation and diarrhea of the rats) or mortality in the investigated groups when compared to the controls, as observed during the 15 days of the acute toxicity experimental study. There was also no weight loss seen in the animals. In the subchronic toxicity test, the rats were free of intoxicating signs and physical changes even when the highest dose of swertiamarin $(500 \mathrm{mg} / \mathrm{kg}$ ) was used throughout the drug dosing period of 50 days. Additionally, no mortality or clinical signs were observed throughout the experimental period. Similarly, Jaishree and Badami ${ }^{5}$ also reported that no acute oral toxicity or mortality was observed in rats administered different doses $(5,50,300$ and $2000 \mathrm{mg} /$ $\mathrm{kg}$ ) of swertiamarin during the 30-day experimental period.

\section{Pharmacokinetics of Swertiamarin}

Very recently, Shi et al ${ }^{89}$ conducted a pharmacokinetic study of swertiamarin in rats, with gentiopicroside used as an internal standard. The half-life of swertiamarin was one hour with low oral bioavailability (5.6-6.7\%). Using the UPLC-Q/TOF-MS/MS technique, Shi et al ${ }^{89}$ discovered six metabolites from the serum, urine, bile and feces of rats. In addition, they elucidated potential metabolic pathways (Figure 10) of swertiamarin in vivo. ${ }^{89}$ Furthermore, Li et al ${ }^{90}$ also performed a pharmacokinetic analysis on the intravenous and oral administration of swertiamarin to rats. Swertiamarin with low absolute bioavailability $(10.3 \%)$ showed rapid absorption and elimination. Swertiamarin $(25 \mathrm{mg} / \mathrm{kg}$, P.O.) was promptly and widely distributed throughout the rat tissues. High concentrations $(800 \mathrm{ng} / \mathrm{mL})$ in the liver and kidney were found, indicating that swertiamarin might have been absorbed in the liver and eliminated by the kidney.
"Qing Ye Dan" is a well-known herb that contains swertiamarin and has been widely used to treat viral hepatitis in China. A liquid chromatography mass spectrometry (LC-MS)/MS method was developed to determine the levels of swertiamarin in rat plasma in three different administration methods: oral administration of swertiamarin alone, Qing Ye Dan tablets and coadministration of swertiamarin $(20 \mathrm{mg} / \mathrm{kg})$ with oleanolic acid. The results demonstrated that pharmacokinetic parameters such as the maximum concentration $\left(\mathrm{C}_{\max }\right)$, area under the curve $\left(\mathrm{AUC}_{0-\infty}\right)$, volume of distribution and clearance were significantly different among the three formulations. The data further indicated that oleanolic acid and other ingredients present in Qing Ye Dan tablets may affect the pharmacokinetic behavior of swertiamarin. ${ }^{91}$

Next, Wu et $\mathrm{al}^{15}$ also investigated swertiamarin $(200 \mathrm{mg} / \mathrm{kg})$ metabolism in rats by using LC-MS after oral administration. LC-MS detected the presence of erythrocentaurin, with a maximum plasma concentration of $425.8 \pm 127.6 \mathrm{ng} / \mathrm{mL}$ at approximately $2 \mathrm{~h}$ following administration. The formation of erythrocentaurin was due to the hydrolysis of swertiamarin by bacterial $\beta$ glucosidase to aglycone. In addition, swertiamarin was also metabolized to dihydroisocoumarin and some alkaloidal compounds in vivo, which may contribute to its pharmacological effect. ${ }^{15}$

At present, only a few studies have been published on the pharmacokinetics of swertiamarin; thus, it should be thoroughly investigated, and its metabolites and mechanisms should be further examined in a detailed and systematic manner.

\section{Cosmetics and Pharmaceutical}

\section{Formulations of Swertiamarin}

Bezivin $^{92}$ studied and patented the cosmetic uses of swertiamarin. The patent claimed that swertiamarin or swertiamarin-enriched plant extract may be used to stimulate the formation or regeneration of the epidermis and/or to stimulate the metabolism of the dermis. ${ }^{92}$ In addition, based on the evidence obtained from clinical studies it is also mentioned that swertiamarin or swertiamarin-enriched plant extract may be used as an anti-aging agent, an antiwrinkle agent, a skin complexation unifying agent, an antiredness agent or as a skin-smoothing agent. ${ }^{92}$ Another study by Muhamad Ibrahim et $\mathrm{al}^{93}$ reported that swertiamarin formulated herbal ointment, which was tested in experimental animal models, showed significant wound 


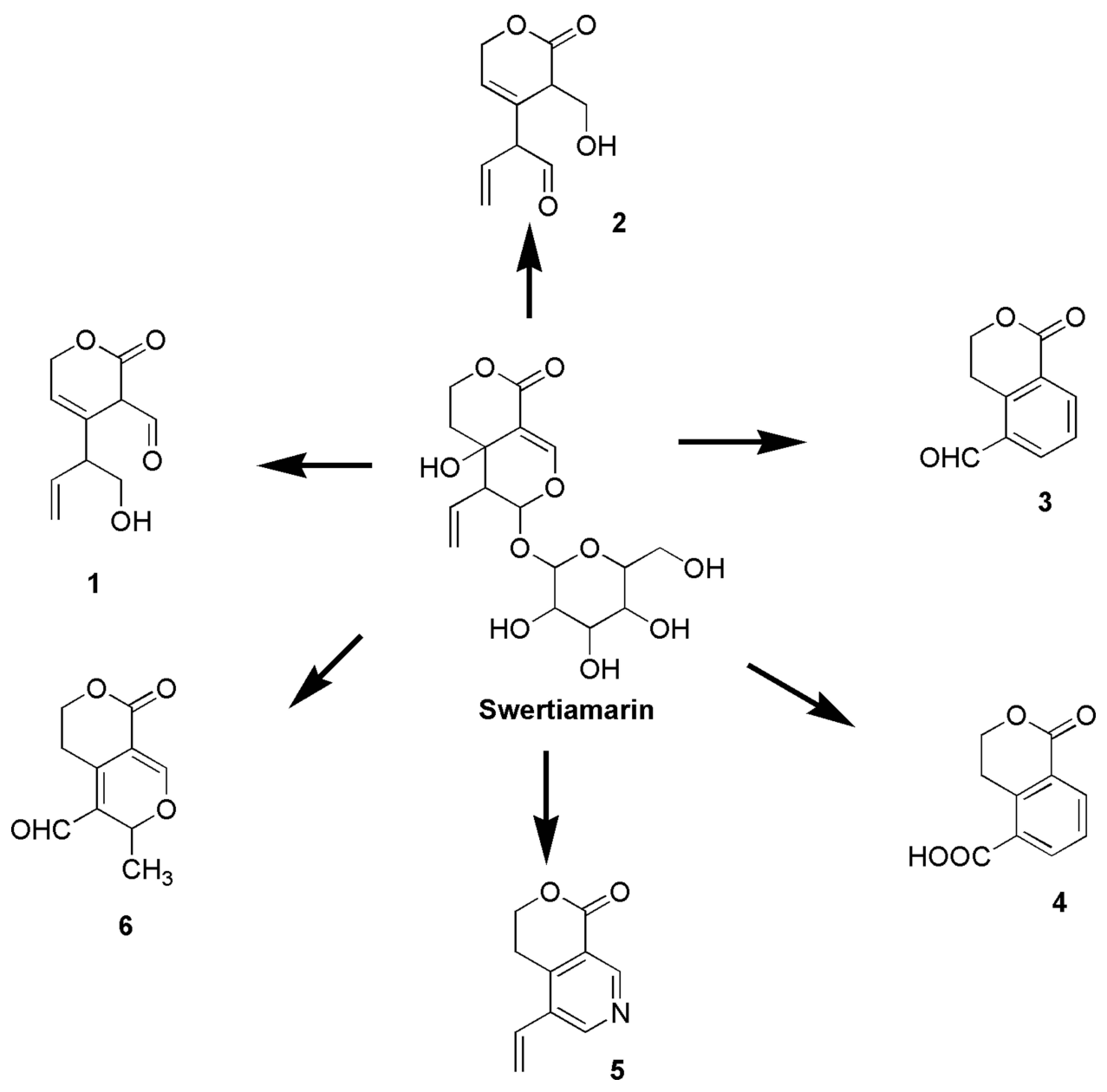

Figure 10 Metabolic pathways of swertiamarin. 4,4a,5,6-Tetrahydro-4a-hydroxy-6-(tetrahydro-3,4,5-trihydroxy-6-(hydroxymethyl)-2H-pyran-2-yloxy)-5-vinylpyrano[3,4-c] pyran-(3H)-one (Swertiamarin). 3,6-Dihydro-4-(I-hydroxybut-3-en-2-yl)-2-oxo-2H-pyran-3-carbaldehyde (I), 2-(3,6-dihydro-3-(hydroxymethyl)-2-oxo-2H-pyran-4-yl)but3-enal (2), I-oxoisochroman-5-carbaldehyde (3), I-oxoisochroman-5-carboxylic acid (4), 3,4-dihydro-5-vinylpyrano[3,4-c]pyridin-I-one (5), and 3,5,6,8-tetrahydro3-methyl-8-oxopyrano[3,4-c] pyran-4-carbaldehyde (6) are the metabolites of swertiamarin. ${ }^{89}$

healing activity. The antiaging properties, as well as other pharmacological activities of swertiamarin, make it a promising element that can be developed by the pharmaceutical industry and established in cosmetic products.

\section{Possible Structural Modifications in Swertiamarin}

It is currently still unclear whether the bioactivities of swertiamarin improve if it undergoes structural modifications. Various analogs of swertiamarin were synthesized (Figure 11) using different chemical reactions, such as fluorination, esterification and epoxidation. ${ }^{94}$ It is clear that the reactions are mainly possible with the glycan hydroxyl groups of swertiamarin. A number of potent antidiabetic swertiamarin derivatives have been developed against 3-hydroxy-3-methyl-glutarylCoA reductase (HMG CoA reductase), and their docking studies have shown markedly similar significant affinity binding modes compared to swertiamarin and its well-known atorvastatin and simvastatin ligands. ${ }^{95}$ Gentianine, a swertiamarin metabolite, revealed increased adipogenesis through upregulation of the protein-encoding genes involved in adipogenesis. ${ }^{66}$ In addition, the synthesized acetylated and propionated swertiamarin derivatives also demonstrated greater antiadipogenic activity than swertiamarin in 3T3-L1 preadipocyte cells. ${ }^{66}$ The in silico design of more potent swertiamarin derivatives can be used as a path toward novel drug discovery and development. Nevertheless, in vitro and in vivo studies should be conducted in the future to confirm the safety and efficacy of all semisynthetic derivatives of swertiamarin. Additionally, more studies relating to its structure-activity relationship (SAR) are warranted in the future to obtain several other novel compounds that can be derived from swertiamarin. 
<smiles>C=C[C@H]1[C@H](O[C@@H]2OC(CF)[C@@H](F)[C@H](F)[C@H]2F)OC=C2C(=O)OCC[C@]21O</smiles>

Flourination<smiles>CO[C@@H]1[C@H](OC(C)=O)[C@@H](COC(C)=O)O[C@H](O[C@@H]2OC=C3C(=O)OCC[C@]3(O)[C@H]2CCO)[C@@H]1OC(C)=O</smiles>

3

Hydroboration<smiles>C=C[C@H]1[C@H](O[C@@H]2OC(CO)[C@@H](O)[C@H](O)[C@H]2O)OC=C2C(=O)OCC[C@]21O</smiles>

Swertiamarin (1)

m-chloroperbenzoic acid

\section{Esterification}<smiles>C=C[C@H]1[C@H](O[C@@H]2OC(COC(C)=O)[C@@H](OC(C)=O)[C@H](OC(C)=O)[C@H]2OC(C)=O)OC=C2C(=O)OCC[C@]21O</smiles>

2<smiles>C=C[C@H]1[C@H](O[C@@H]2OC(COC(C)=O)[C@@H](OC(C)=O)[C@H](OC(C)=O)[C@H]2OC)OC=C2C(F)(F)OCC[C@]21O</smiles>

5<smiles>C=C[C@H]1[C@H](OC(=O)c2cccc(Cl)c2)OC=C2C(=O)OCC[C@@]2(O)[C@@H]1C=C</smiles>

Figure I I Structural modifications of swertiamarin.

Notes: Esterification of glycan hydroxyl groups present in swertiamarin (I) using acetic anhydride in the presence of pyridine and dimethylformamide (DMF) to form swertiamarin tetraacetate (2). A hydroboration reaction was performed using trihydridoboron $\left(\mathrm{BH}_{3}\right)$-tetrahydrofuran $(\mathrm{THF})$, hydrogen peroxide $\left(\mathrm{H}_{2} \mathrm{O} \mathrm{O}_{2}\right)$ and $\mathrm{NaOH}$ to yield 3. A nucleophilic fluorination reaction was performed to attach the fluorine atom at the positions of a hydroxyl group in the glycan part of I using diethylaminosulphurtrifluoride (DAST) reagent to yield 4. Fluorination also takes place at the lactone group at (C-8) position 2 by using DAST reagent to form 5 . When I and 2 were treated with m-chloroperbenzoic acid (mCPBA) for epoxidation, unexpected product 6 was formed due to anchimeric assistance. ${ }^{94}$

\section{Drug-Likeness Properties of Swertiamarin}

Regarding the physicochemical properties of swertiamarin, we investigated the results by applying Lipinski's rule of five to determine its drug-likeness appearance. ${ }^{46}$ According to Lipinski's rule of five, any drug-like compound should have 1) a molecular weight of $\leq 500 ; 2$ ) a partition coefficient $(\log P)$-value of $\leq 5$; 3$) \mathrm{H}$-bond donors $\leq 5$; 4) H-bond acceptors $\leq 10$; and 5 ) rotatable bonds $\leq 10$. Since compounds that do not breach Lipinski's rule of five may have enhanced 
folding, polarity, and molecular size, it is deemed that druglike molecules have the expected pharmaceutical properties. ${ }^{96}$ Since Lipinski's rule of five is an effective and valid guide for forecasting the potential for chemical compounds to be effective as orally administered drugs, the aim of the suggested method was merely to deliver primary evidence defining the potential physical properties of the compounds, rather than serving as rigid screening criteria by itself. Overall, based on the findings, swertiamarin meets all of Lipinski's rule of five for drug-likeness properties (Table 1), indicating that it may be a potential therapeutic agent to combat various diseases.

\section{Possible Cellular and Molecular Targets of Swertiamarin}

Swertiamarin interacts either directly or indirectly with various molecular targets in addition to modulating their activity and function. Swertiamarin's cellular and molecular targets are outlined in Figure 12, with some effects hypothesized to be dose-dependent. Depending on its dose or concentration, swertiamarin, for instance, both increases and decreases the proteins and markers of apoptosis. In fact, further investigations using a wide range of doses are required to investigate the dose-dependent effects of swertiamarin to understand the exact mechanism.

\section{Challenges and the Need for Clinical Research}

A variety of beneficial properties related to human health have been reported for swertiamarin, including analgesic, anti-inflammatory, antiarthritis, hepatoprotective, antidiabetic, antioxidant, antimicrobial, anticancer, neuroprotective and gastroprotective effects. However, although it has been considered to be a compound with a wide range of pharmacological activities, to the best of our knowledge,

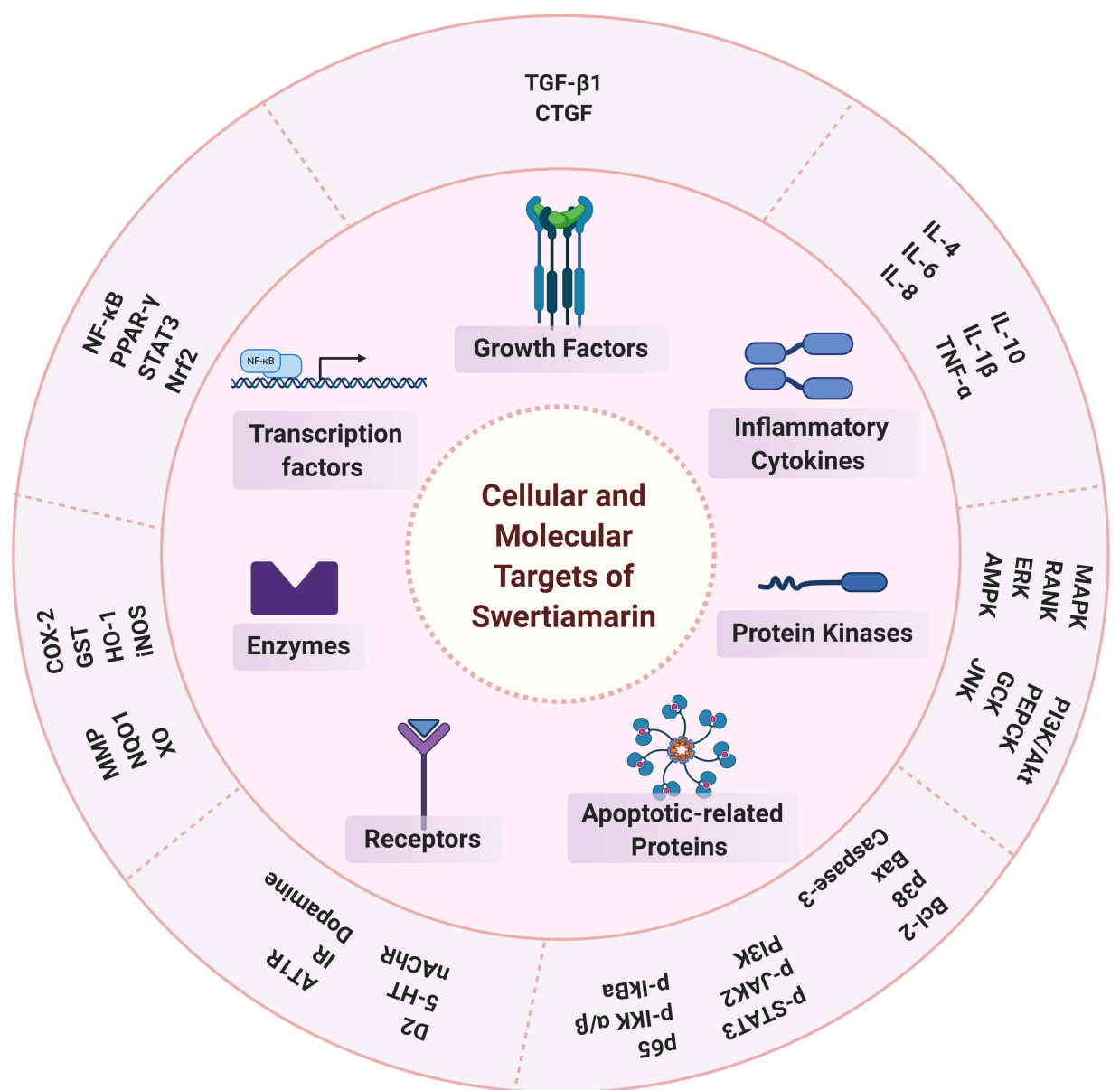

Figure 12 Cellular and molecular targets of swertiamarin.

Note: The figure was created with the support of https://biorender.com under the paid subscription. 
there is no clinical research reported so far. Therefore, clinical research on the pharmacology and pharmacokinetics of swertiamarin is required, since most of these effects have only been shown in preclinical experiments. Based on the studies reported on swertiamarin, swertiamarin will eventually result in changes in the body at the level of molecules, cells, tissues and/or organs, along with its disease ameliorative effects when administered to animals or humans. Moreover, swertiamarin has become a favored choice for current research due to its multidimensional therapeutic potential and can be an outstanding novel candidate for novel drug design and the development of new derivatives with improved pharmacological and therapeutic potential. Thus, clinical trials on swertiamarin are required to evaluate the pharmacodynamic parameters caused by its physicochemical properties, including its bioavailability, half-life, adverse reactions and toxicity. In addition, clinical trials should be performed to enable the wide applicability of effective bioactive compounds.

\section{Conclusion and Future Perspectives}

This review highlights the distribution of swertiamarin in medicinal plants and its isolation, structural characterization, physicochemical properties, and biosynthesis. We have also provided scientific updates on its pharmacology and therapeutic potential. Swertiamarin has gained recognition for its many health benefits that mainly appear to function through its antioxidant and anti-inflammatory mechanisms. Accumulating evidence provided by various preclinical studies has shown that swertiamarin can help to treat multiple diseases, based on its analgesic, antiinflammatory, antiarthritic, hepatoprotective, antidiabetic, antioxidant, antimicrobial, anticancer, neuroprotective and gastroprotective properties. Swertiamarin can be considered a powerful antioxidant along with multifaceted molecular targets, such as the NF- $\mathrm{kB}$, MAPK/TGF- $\beta 1$, AMPK, $\mathrm{PI} 3 \mathrm{~K} / \mathrm{Akt}$, Nrf2-HO-1, and PPAR $\gamma$ signaling pathways, in various pathological conditions. It can modulate multiple signaling pathways, numerous molecular targets, and gene expression. The metabolic processes and pharmacokinetics of swertiamarin in vivo have mostly been investigated in experimental animal models but have not been studied to date in the human body; thus, clinical studies are needed. Therefore, following a thorough analysis of its toxicities, detailed studies on swertiamarin are required to enhance its bioavailability and to develop novel dosage forms. In addition, novel delivery strategies, including nanoparticles, liposomes, phospholipid complexes, microemulsions or polymer micelles, are suggested to boost the pharmacokinetic properties and improve its oral bioavailability, which can help pave the way for more applicative studies and clinical trials in the near future. It is hoped that this review will stimulate further investigations on swertiamarin related to its pharmacokinetics, pharmacodynamics, and clinical and SAR studies. All these additional parameters will accelerate the development and utilization of swertiamarin as a promising drug candidate in the near future.

\section{Highlights}

- Swertiamarin is a seco-iridoid glycoside that exhibits a broad spectrum of pharmacological properties.

- It meets Lipinski's rule of five for drug-likeness properties.

- It is biosynthesized by mevalonate or nonmevalonate pathways followed by the seco-iridoid pathway.

- This review emphasizes the distribution, isolation, characterization, physicochemical properties, biosynthetic pathways, toxicity profiles, biological properties, metabolic pathways, pharmacokinetics and structural modifications of swertiamarin.

- This review accelerates the development and utilization of swertiamarin as a promising natural lead for new drug discovery in the near future.

\section{Abbreviations}

10-HGO, 10-hydroxygeraniol oxidoreductase; 5-HT, 5-hydroxytryptamine; 7DLS, 7-deoxyloganetic acid synthase; AACT, Acetoacetyl-CoA thiolase; Akt, Serine/threonine protein kinase; ALT, Alanine aminotransferase; AMPK, Adenosine monophosphate-activated protein kinase; AST, Aspartate transaminase; AT1R, Angiotensin II type 1 receptor; $\mathrm{BH}_{3}$, Trihydridoboron; $\mathrm{CCl}_{4}$, Carbon tetrachloride; CGBVS, Chemical genomics-based virtual screening; CMK, 4-diphosphocytidyl-2-Cmethyl-D-erythritol kinase; CMS, 4-diphosphocytidyl-2-C-methyl-D-erythritol synthase; Con A, Concanavalin A; CYP, Cytochrome; DAST, Diethylaminosulphurtrifluoride; d-GaIN, Galactosamine; DLGT, 7-deoxyloganetic acid glucosyltransferase; DL7H, 7-deoxyloganic acid 7-hydroxylase; LAMT, Loganic acid O-methyltransferase; SLS, Secologanin synthase; DMAPP, dimethylallyl diphosphate; DMF, Dimethylformamide; DMN, Dimethylnitrosamine; DXR, 1-deoxy-D-xylulose5-phosphate reductoisomerase; DXS, 1-deoxy-D-xylulose5-phosphate synthase; ERK, Extracellular signal-regulated kinase; ESI-MS, Electrospray ionization mass spectrometry; FAS, Fatty acid synthase; FLS, Fibroblast-like synoviocytes; 
FTIR, Fourier-transform Infrared spectroscopy; $\mathrm{G} 10 \mathrm{H}$, Geraniol 10-hydroxylase; GLUT-4, Glucose transporter type 4; $\mathrm{H}_{2} \mathrm{O}_{2}$, Hydrogen peroxide; HA, Hemagglutinating antibody; HbA1c, Hemoglobin A1c; HDL, High-density lipoprotein; HDR, 1-hydroxy-2-methyl-2-butenyl 4-diphosphate reductase; HDS, 1-hydroxy-2-methyl-2-butenyl 4-diphosphate synthase; HepG2, Human liver cancer cell line; HMGCoA, 3-hydroxy-3-methyl-glutaryl-coenzyme A reductase; HMGR, Hydroxymethyglutaryl-CoA reductase; HMGS, Hydroxymethyglutaryl-CoA synthase; HSC, Hepatic stellate cells; i.p., intraperitoneal; $\mathrm{IC}_{50}$, Half maximal inhibitory concentration; IDI, Isopentenyl diphosphate isomerase; IL, Interleukin; iNOS, Inducible nitric oxide synthase; IRS, Iridoid synthase; LDL, Low-density lipoprotein; LPS, Lipopolysaccharide; MAPK, Mitogen-activated protein kinase; MCAO, Multi-conjugate adaptive optics; mCPBA, m-chloroperbenzoic acid; MDA, Malondialdehyde; MECS, 2-C-methyl-D-erythritol 2,4-cyclodiphosphate synthase; MMT, Manual muscle testing; mRNA, Messenger RNA; MVD, Mevalonate 5-diphosphate decarboxylase; MVK, Mevalonate kinase; NAFLD, Nonalcoholic fatty liver disease; NF- $\kappa B$, Nuclear factor kappa-light-chain-enhancer of activated B cells; NMR, Nuclear magnetic resonance spectroscopy; NQO1, NAD(P)H, quinone oxidoreductase-1; Nrf2, Nuclear factor E2-related factor 2; P.O., Oral administration; PI3K, Phosphatidylinositol 3-kinase; PMK, Mevalonate 5-phosphate kinase; PPAR- $\alpha$, Peroxisome proliferatoractivated receptor alpha; PTZ, Pentylenetetrazole; RANKL, Osteoclastogenic mediator; RAS, Renin-angiotensin system; s.c., Subcutaneous; SAR, Structure activity relationship; SRB, Sulfate reducing bacteria; SREBP-1, Sterol-regulatory element-binding protein-1; STZ, Streptozotocin; TG, Triglyceride; TGF- $\beta 1$, Transforming growth factor- $\beta 1$; THF, Tetrahydrofuran; TUNEL, Terminal deoxynucleotidyl transferase dUTP nick end labelling; UV, Ultraviolet; XO, xanthine oxidase.

\section{Consent for Publication}

The final version of the manuscript was reviewed by all the authors and consented to its submission.

\section{Acknowledgments}

The authors thank Universiti Kuala Lumpur Royal College of Medicine Perak, Malaysia, for providing necessary facilities and resources to complete this study for publication.

\section{Author Contributions}

N.S.M.F., and M.S. conceived the idea, designed, collected the literature, interpreted the data, analyzed the data, drafted and revised the manuscript. All the authors have made noteworthy contributions to the study design, data collection, review and interpretation; have engaged in the drafting or revision of the article; have agreed to submit to the current journal; have given final approval of the version to be published; and have agreed to be responsible for all aspects of the work.

\section{Funding}

There is no funding to report.

\section{Disclosure}

The authors report no conflicts of interest in this work.

\section{References}

1. Bonam SR, Wu YS, Tunki L, et al. What has come out from phytomedicines and herbal edibles for the treatment of cancer? Chem Med Chem. 2018;13(18):1854-1872. doi:10.1002/ cmdc. 201800343

2. Ramakrishnan P, Loh WM, Gopinath SC, et al. Selective phytochemicals targeting pancreatic stellate cells as new anti-fibrotic agents for chronic pancreatitis and pancreatic cancer. Acta Pharm Sin B. 2020;10(3):399-413. doi:10.1016/j.apsb.2019.11.008

3. Anwar MMA, Aftab K, Aftab K, Aftab K. Enicostema littorale: a new source of swertiamarin. Pak J Pharm Sci. 1996;9(1):29-35.

4. Saranya R, Thirumalai T, Hemalatha M, et al. Pharmacognosy of Enicostemma littorale: a review. Asian Pac J Trop Biomed. 2013;3 (1):79-84. doi:10.1016/S2221-1691(13)60028-3

5. Jaishree V, Badami S. Antioxidant and hepatoprotective effect of swertiamarin from Enicostemma axillare against D-galactosamine induced acute liver damage in rats. J Ethnopharmacol. 2010;130 (1):103-106. doi:10.1016/j.jep.2010.04.019

6. Maroo J, Vasu V, Gupta S. Dose dependent hypoglycemic effect of aqueous extract of Enicostemma littorale Blume in alloxan induced diabetic rats. Phytomedicine. 2003;10(2-3):196-199. doi:10.1078/ 094471103321659933

7. Piatczak E, Krolicka A, Wysokinska H. Genetic transformation of Centaurium erythraea Rafn by Agrobacterium rhizogenes and the production of secoiridoids. Plant Cell Rep. 2006;25(12):1308-1315. doi:10.1007/s00299-006-0155-0

8. Abe N, Nakano Y, Shimogomi A, et al. A new flavonol triglycoside from Eustoma grandiflorum. Nat Prod Commun. 2016;11 (7):963-964.

9. Jonville M-C, Capel M, Frédérich M, et al. Fagraldehyde, a secoiridoid isolated from Fagraea fragrans. $J$ Nat Prod. 2008;71 (12):2038-2040. doi:10.1021/np800291d

10. Tan R, Hu J, Dong L, et al. Two new secoiridoid glycosides from Gentiana algida. Planta Med. 1997;63(06):567-569. doi:10.1055/ s-2006-957770

11. Zhou L, Li X-K, Miao F, et al. Further studies on the chemical constituents of Chinese folk medicine Gentiana apiata NE Br. $J$ Asian Nat Prod Res. 2009;11(4):345-351. doi:10.1080/ 10286020902819814 
12. Mihailovic V, Matic S, Mišic D, et al. Chemical composition, antioxidant and antigenotoxic activities of different fractions of Gentiana asclepiadea L. roots extract. EXCLI J. 2013;12:807.

13. Wanga Y, Ahmadc B, Duana B, et al. Chemical and genetic comparative analysis of Gentiana crassicaulis and Gentiana. Chem Biodivers. 2016;13:1-6. doi:10.1002/cbdv.201400363

14. Mihailović V, Katanić J, Mišić D, et al. Hepatoprotective effects of secoiridoid-rich extracts from Gentiana cruciata L. against carbon tetrachloride induced liver damage in rats. Food Funct. 2014;5 (8):1795-1803. doi:10.1039/C4FO00088A

15. Wu X, Tang S, Jin Y, et al. New analytical method for the study of metabolism of swertiamarin in rats after oral administration by UPLC-TOF-MS following DNPH derivatization. Biomed Chromatogr. 2015;29(8):1184-1189. doi:10.1002/bmc.3406

16. Chueh F-S, Chen -C-C, Sagare AP, et al. Quantitative determination of secoiridoid glucosides in in vitro propagated plants of Gentiana davidii var. formosana by high performance liquid chromatography. Planta Med. 2001;67(1):70-73. doi:10.1055/s-2001-10622

17. Çaliş İ, Rüegger H, Chun Z, et al. Secoiridoid glucosides isolated from Gentiana gelida. Planta Med. 1990;56(4):406-409. doi:10.1055/ s-2006-960995

18. Ghazanfar K, Mubashir K, Dar SA, et al. Gentiana kurroo Royle attenuates the metabolic aberrations in diabetic rats; Swertiamarin, swertisin and lupeol being the possible bioactive principles. J Complement Integr Med. 2017;14(3):3. doi:10.1515/jcim-2017-0002

19. Zhang X, Zhang H, Ji L. Simultaneous determination of four constituents in wild Gentiana lawrencei from Qinghai province by RP-HPLC. China J Chinese Mat Med. 2009;34(22):2884-2886.

20. Kesavan R, Potunuru UR, Nastasijević B, et al. Inhibition of vascular smooth muscle cell proliferation by Gentiana lutea root extracts. PLoS One. 2013;8(4):e61393. doi:10.1371/journal.pone.0061393

21. Zhang J, Tian Z, Lou Z. Simultaneous determination of five bitter secoiridoid glycosides in nine Chinese Gentiana species used as the Chinese drug "long dan" by high performance liquid chromatography. Acta Pharm Sin. 1991;26(11):864-870.

22. Suyama Y, Kurimoto S-I, Kawazoe K, et al. Rigenolide A, a new secoiridoid glucoside with a cyclobutane skeleton, and three new acylated secoiridoid glucosides from Gentiana rigescens Franch. Fitoterapia. 2013;91:166-172. doi:10.1016/j.fitote.2013.08.006

23. Kshirsagar PR, Pai SR, Nimbalkar MS, et al. RP-HPLC analysis of seco-iridoid glycoside swertiamarin from different Swertia species. Nat Prod Res. 2016;30(7):865-868. doi:10.1080/ 14786419.2015.1071366

24. Luo Y, Nie R. Studies on iridoid glycosides from Swertia angustifolia. Acta Pharm Sin. 1992;27(2):125.

25. Lad H, Bhatnagar D. Amelioration of oxidative and inflammatory changes by Swertia chirayita leaves in experimental arthritis. Inflammopharmacology. 2016;24(6):363-375. doi:10.1007/s10787016-0290-3

26. Mahendran G, Thamotharan G, Sengottuvelu S, et al. RETRACTED: anti-diabetic activity of Swertia corymbosa (Griseb.) Wight ex C.B. Clarke aerial parts extract in streptozotocin induced diabetic rats. J Ethnopharmacol. 2014;151(3):1175-1183. doi:10.1016/j. jep.2013.12.032

27. Cao T-W, Geng C-A, Ma Y-B, et al. Chemical constituents of Swertia mussotii and their anti-hepatitis B virus activity. Fitoterapia. 2015;102:15-22. doi:10.1016/j.fitote.2015.01.020

28. Wang -S-S, Zhao W-J, Han X-W, et al. Two new iridoid glycosides from the Tibetan folk medicine Swertia franchetiana. Chem Pharm Bull. 2005;53(6):674-676. doi:10.1248/cpb.53.674

29. Jiang FQ, Zhang XM, Ma Y, et al. Chemical constituents of Swertia hispidicalyx. J Chinese Mat Med. 2011;36(16):2215-2218.

30. Rai A, Nakamura M, Takahashi H, et al. High-throughput sequencing and de novo transcriptome assembly of Swertia japonica to identify genes involved in the biosynthesis of therapeutic metabolites. Plant Cell Rep. 2016;35(10):2091-2111. doi:10.1007/s00299-016-2021-z
31. He K, Cao TW, Wang HL, et al. Chemical constituents of Swertia kouitchensis Franch. China J Chinese Mat Med. 2015;40(19):3811-3817.

32. Saeidnia S, Ara L, Hajimehdipoor H, et al. Chemical constituents of Swertia longifolia Boiss. with $\alpha$-amylase inhibitory activity. Res Pharm Sci. 2016;11(1):23.

33. Kikuzaki H, Kawasaki Y, Kitamura S, et al. Secoiridoid Glucosides from Swertia mileensis. Planta Med. 1996;62(01):35-38. doi:10.1055/s-2006-957792

34. Tian C, Zhang T, Wang L, et al. The hepatoprotective effect and chemical constituents of total iridoids and xanthones extracted from Swertia mussotii Franch. J Ethnopharmacol. 2014;154(1):259-266. doi:10.1016/j.jep.2014.04.018

35. He K, Cao T, Wang H, et al. Chemical constituents of Swertia patens. China J Chinese Mat Med. 2015;40(20):4012.

36. Huang W-Z, Xu C-H, Zhou D-C, et al. Determination of iridoids and triterpenes in herb of Swertia pseudochinesis by RP-HPLC. China J Chinese Mat Med. 2007;32(23):2494-2496.

37. Tan P, Liu Y, Hou C. Swertiapunimarin from Swertia punicea Hemsl. Acta Pharm Sin. 1993;28(7):522-525.

38. Nwafor PA, Abia GO, Bankhede HK. Antipyretic and antimalarial activities of crude leaf extract and fractions of Enicostema littorale. Asian Pac J Trop Dis. 2012;2(6):442-447. doi:10.1016/S22221808(12)60097-8

39. Takate S, Pokharkar R, Chopade V, et al. Hepatoprotective activity of the ethyl acetate extract of Launaea intybacea (jacq) beauv in paracetamol induced hepato-toxicity in albino rats. Int J Pharm Sci Rev Res. 2010;1(2):72-74.

40. Patel N, Tyagi RK, Tandel N, et al. The molecular targets of swertiamarin and its derivatives confer anti-diabetic and anti-hyperlipidemic effects. Curr Drug Targets. 2018;19(16):1958-1967. doi:10.2174/ 1389450119666180406113428

41. Jaishree V, Badami S, Kumar MR, et al. Antinociceptive activity of swertiamarin isolated from Enicostemma axillare. Phytomedicine. 2009;16(2-3):227-232. doi:10.1016/j.phymed.2008.09.010

42. Vishwakarma S, Rajani M, Bagul M, et al. A rapid method for the isolation of swertiamarin from Enicostemma littorale. Pharm Biol. 2004;42(6):400-403. doi:10.1080/13880200490885095

43. Kakarla RK, Rajendran M, Paulraj B, et al. Isolation and characterization of Swertiamarin from aerial parts of Enicostemma littorale Blume. Int J Bioassays. 2019;8(1):5712-5715.

44. Rana V. Separation and identification of swertiamarin from Enicostema axillare Lam. raynal by centrifugal partition chromatography and nuclear magnetic resonance-mass spectrometry. $J$ Pharm Sci Emerg Drugs. 2014;1:2.

45. NIH. Swertiamarin. Vol. 2020. 2020.

46. Lipinski CA, Lombardo F, Dominy BW, et al. Experimental and computational approaches to estimate solubility and permeability in drug discovery and development settings. Adv Drug Deliv Rev. 1997;23(1-3):3-25. doi:10.1016/S0169-409X(96)00423-1

47. Miettinen K, Dong L, Navrot N, et al. The seco-iridoid pathway from Catharanthus roseus. Nat Commun. 2014;5(1):1-12.

48. Oudin A, Mahroug S, Courdavault V, et al. Spatial distribution and hormonal regulation of gene products from methyl erythritol phosphate and monoterpene-secoiridoid pathways in Catharanthus roseus. Plant Mol Biol. 2007;65(1-2):13-30. doi:10.1007/s11103-007-9190-7

49. Loyola-Vargas VM, Galaz-ávalos RM, Kú-Cauich R. Catharanthus biosynthetic enzymes: the road ahead. Phytochem Rev. 2007;6 (2):307-339. doi:10.1007/s11101-007-9064-2

50. Collu G, Unver N, Peltenburg-Looman AM, et al. Geraniol 10-hydroxylase 1, a cytochrome P450 enzyme involved in terpenoid indole alkaloid biosynthesis. FEBS Lett. 2001;508(2):215-220. doi:10.1016/S0014-5793(01)03045-9

51. Irmler S, Schröder G, St-Pierre B, et al. Indole alkaloid biosynthesis in Catharanthus roseus: new enzyme activities and identification of cytochrome P450 CYP72A1 as secologanin synthase. Plant J. 2000;24(6):797-804. doi:10.1046/j.1365-313x.2000.00922.x 
52. Murata J, Roepke J, Gordon H, et al. The leaf epidermome of Catharanthus roseus reveals its biochemical specialization. Plant Cell. 2008;20(3):524-542. doi:10.1105/tpc.107.056630

53. Zhu X, Zeng X, Sun C, et al. Biosynthetic pathway of terpenoid indole alkaloids in Catharanthus roseus. Front Med. 2014;8 (3):285-293. doi:10.1007/s11684-014-0350-2

54. Zhang M, Ma X, Xu H, et al. A natural AKT inhibitor swertiamarin targets AKT-PH domain, inhibits downstream signaling, and alleviates inflammation. FEBS J. 2020;287(9):1816-1829. doi:10.1111/febs.15112

55. Saravanan S, Islam VH, Babu NP, et al. Swertiamarin attenuates inflammation mediators via modulating NF- $\mathrm{KB} / \mathrm{I} \kappa \mathrm{B}$ and JAK2/ STAT3 transcription factors in adjuvant induced arthritis. Eur J Pharm Sci. 2014;56:70-86. doi:10.1016/j.ejps.2014.02.005

56. Saravanan S, Islam VH, Thirugnanasambantham K, et al. Swertiamarin ameliorates inflammation and osteoclastogenesis intermediates in IL-1 $\beta$ induced rat fibroblast-like synoviocytes. Inflamm Res. 2014;63(6):451-462. doi:10.1007/s00011-014-0717-5

57. Saravanan S, Pandikumar P, Babu NP, et al. In vivo and in vitro immunomodulatory potential of swertiamarin isolated from Enicostema axillare (Lam.) A. Raynal that acts as an anti-inflammatory agent. Inflammation. 2014;37(5):1374-1388. doi:10.1007/s10753-014-9862-9

58. Hairul-Islam M, Saravanan S, Thirugnanasambantham K, et al. Swertiamarin, a natural steroid, prevent bone erosion by modulating RANKL/RANK/OPG signaling. Int Immunopharmacol. 2017;53:114-124. doi:10.1016/j.intimp.2017.10.022

59. Vaijanathappa J, Badami S. Antiedematogenic and free radical scavenging activity of swertiamarin isolated from Enicostemma axillare. Planta Med. 2009;75(01):12-17. doi:10.1055/s-0028-1088333

60. Yang Y, Li J, Wei C, et al. Amelioration of nonalcoholic fatty liver disease by swertiamarin in fructose-fed mice. Phytomedicine. 2019;59:152782. doi:10.1016/j.phymed.2018.12.005

61. Zhang Q, Chen K, Wu T, et al. Swertiamarin ameliorates carbon tetrachloride-induced hepatic apoptosis via blocking the PI3K/Akt pathway in rats. Korean J Physiol Pharmacol. 2019;23(1):21-28. doi:10.4196/kjpp.2019.23.1.21

62. Wu T, Li J, Li Y, et al. Antioxidant and hepatoprotective effect of swertiamarin on carbon tetrachloride-induced hepatotoxicity via the Nrf2/HO-1 pathway. Cell Physiol Biochem. 2017;41(6):2242-2254. doi:10.1159/000475639

63. Li S, Wang Q, Tao Y, et al. Swertiamarin attenuates experimental rat hepatic fibrosis by suppressing angiotensin II-angiotensin type 1 receptor-extracellular signal-regulated kinase signaling. $J$ Pharmacol Exp Ther. 2016;359(2):247-255. doi:10.1124/ jpet.116.234179

64. Phoboo S, Pinto MDS, Barbosa ACL, et al. Phenolic-linked biochemical rationale for the antidiabetic properties of Swertia chirayita (Roxb. ex Flem.) Karst. Phytother Res. 2013;27(2):227-235. doi: $10.1002 /$ ptr.4714

65. Wani BA, Ramamoorthy D, Rather MA, et al. Induction of apoptosis in human pancreatic MiaPaCa-2 cells through the loss of mitochondrial membrane potential $(\Delta \Psi \mathrm{m})$ by Gentiana kurroo root extract and LC-ESI-MS analysis of its principal constituents. Phytomedicine. 2013;20(8-9):723-733. doi:10.1016/j.phymed.2013.01.011

66. Vaidya H, Goyal RK, Cheema SK. Antidiabetic activity of swertiamarin is due to an active metabolite, gentianine, that upregulates PPAR- $\gamma$ gene expression in 3T3-L1 cells. Phytother Res. 2013;27 (4):624-627. doi:10.1002/ptr.4763

67. Vaidya H, Giri S, Jain M, et al. Decrease in serum matrix metalloproteinase-9 and matrix metalloproteinase-3 levels in Zucker $\mathrm{fa} / \mathrm{fa}$ obese rats after treatment with swertiamarin. Exp Clin Cardiol. 2012;17(1):12.

68. Vaidya H, Prajapati A, Rajani M, et al. Beneficial effects of swertiamarin on dyslipidaemia in streptozotocin-induced type 2 diabetic rats. Phytother Res. 2012;26(8):1259-1261. doi:10.1002/ptr.3708
69. Dhanavathy G. Immunohistochemistry, histopathology, and biomarker studies of swertiamarin, a secoiridoid glycoside, prevents and protects streptozotocin-induced $\beta$-cell damage in Wistar rat pancreas. J Endocrinol Investig. 2015;38(6):669-684. doi:10.1007/s40618-015-0243-5

70. Patel TP, Soni S, Parikh P, et al. Swertiamarin: an active lead from Enicostemma littorale regulates hepatic and adipose tissue gene expression by targeting PPAR- $\gamma$ and improves insulin sensitivity in experimental NIDDM rat model. J Evid Based Complementary Altern Med. 2013;2013.

71. Sonawane RD, Vishwakarma SL, Lakshmi S, et al. Amelioration of STZ-induced type 1 diabetic nephropathy by aqueous extract of Enicostemma littorale Blume and swertiamarin in rats. Mol Cell Biochem. 2010;340(1-2):1-6. doi:10.1007/s11010-010-0393-x

72. Ahamad J, Hassan N, Amin S, et al. Swertiamarin contributes to glucose homeostasis via inhibition of carbohydrate metabolizing enzymes. $J$ Nat Med. 2016;4:125-130.

73. Patel TP, Rawal K, Soni S, et al. Swertiamarin ameliorates oleic acid induced lipid accumulation and oxidative stress by attenuating gluconeogenesis and lipogenesis in hepatic steatosis. Biomed Pharmacother. 2016;83:785-791. doi:10.1016/j.biopha.2016.07.028

74. Kimura Y, Sumiyoshi M. Effects of Swertia japonica extract and its main compound swertiamarin on gastric emptying and gastrointestinal motility in mice. Fitoterapia. 2011;82(6):827-833. doi:10.1016/j. fitote. 2011.04.008

75. Yao L-S, Tang L-N, Liu C-Q, et al. Study on protective effect and possible mechanisms of swertiamarin against sepsis-induced acute lung injury in rats. Int J Clin Exp Med. 2016;9(7):13445-13455.

76. Pandey T, Smita SS, Mishra A, et al. Swertiamarin, a secoiridoid glycoside modulates $\mathrm{nAChR}$ and $\mathrm{AChE}$ activity. Exp Geront. 2020;138:111010. doi:10.1016/j.exger.2020.111010

77. Deng X-H, Zhang X, Wang J, et al. Anticonvulsant effect of swertiamarin against Pilocarpine-induced seizures in adult male mice. Neurochem Res. 2017;42(11):3103-3113. doi:10.1007/s11064-017-2347-0

78. Devinsky O, Vezzani A, Najjar S, et al. Glia and epilepsy: excitability and inflammation. Trends Neurosci. 2013;36(3):174-184. doi:10.1016/j.tins.2012.11.008

79. Vezzani A, Baram TZ. New roles for interleukin-1 Beta in the mechanisms of epilepsy. Epilepsy Curr. 2007;7(2):45-50. doi:10.1111/j.1535-7511.2007.00165.x

80. Uludag IF, Duksal T, Tiftikcioglu BI, et al. IL-1 $\beta$, IL-6 and IL1Ra levels in temporal lobe epilepsy. Seizure. 2015;26:22-25. doi:10.1016/j.seizure.2015.01.009

81. Vezzani A, Aronica E, Mazarati A, et al. Epilepsy and brain inflammation. Exp Neurol. 2013;244:11-21. doi:10.1016/j.expneurol.2011.09.033

82. Alsharafi WA, Xiao B, Abuhamed MM, et al. Correlation between IL-10 and microRNA-187 expression in epileptic rat hippocampus and patients with temporal lobe epilepsy. Front Cell Neurosci. 2015;9:466. doi:10.3389/fncel.2015.00466

83. Vaijanathappa J, Puttaswamygowda J, Bevanhalli R, et al. Molecular docking, antiproliferative and anticonvulsant activities of swertiamarin isolated from Enicostemma axillare. Bioorg Chem. 2020;94:103428. doi:10.1016/j.bioorg.2019.103428

84. Wang H, Wei W, Lan X, et al. Neuroprotective effect of swertiamain on cerebral ischemia/reperfusion injury by inducing the Nrf2 protective pathway. ACS Chem Neurosci. 2019;10(5):2276-2286. doi:10.1021/acschemneuro.8b00605

85. Tang H, Ke Y, Ren Z, et al. Bioinformatics analysis of differentially expressed genes in hepatocellular carcinoma cells exposed to Swertiamarin. J Cancer. 2019;10(26):6526. doi:10.7150/jca.33666

86. Dubey S, Kallubai M, Subramanyam R. Comparative binding of swertiamarin with human serum albumin and $\alpha-1$ glycoprotein and its cytotoxicity against neuroblastoma cells. J Biomol Struct Dyn. 2019;1-11.

87. Šiler B, Mišić D, Nestorović J, et al. Antibacterial and antifungal screening of Centaurium pulchellum crude extracts and main secoiridoid compounds. Nat Prod Commun. 2010;5 (10):1934578X1000501001. 
88. Dhanavathy G, Jayakumar S. Acute and subchronic toxicity studies of Swertiamarin a lead compound isolated from Enicostemma littorale Blume in wistar rats. Biosci Biotech Res Asia. 2017;14 (1):381-390. doi:10.13005/bbra/2456

89. Shi M, Xiong K, Zhang T, et al. Pharmacokinetics and metabolic profiles of swertiamarin in rats by liquid chromatography combined with electrospray ionization tandem mass spectrometry. J Pharm Biomed. 2020;179:112997. doi:10.1016/j.jpba.2019.112997

90. Li H-L, He J-C, Bai M, et al. Determination of the plasma pharmacokinetic and tissue distributions of swertiamarin in rats by liquid chromatography with tandem mass spectrometry. Drug Res. 2012;62(03):138-144.

91. Xu G-L, Li H-L, He J-C, et al. Comparative pharmacokinetics of swertiamarin in rats after oral administration of swertiamarin alone, Qing Ye Dan tablets and co-administration of swertiamarin and oleanolic acid. J Ethnopharmacol. 2013;149(1):49-54. doi:10.1016/ j.jep.2013.05.016

92. Bezivin C. Cosmetic uses of swertiamarin. Google Patents. 2017.
93. Muhamad Ibrahim A, Nagarajan A, Majeed M. Swertiamarin ointment: a traditional approach in cutaneous wound healing. Asian J Pharm Pharmacol. 2019;5(2):298-309. doi:10.31024/ ajpp.2019.5.2.13

94. Kumar S, Niguram P, Bhat V, et al. Synthesis, molecular docking and ADMET prediction of novel swertiamarin analogues for the restoration of type-2 diabetes: an enzyme inhibition assay. Nat Prod Res. 2020;1-11.

95. Bhatt J, Vaidya $H$, Khanna $\mathrm{V}$, et al. In silico docking studies for designing potent anti-diabetic derivatives of swertiamarin with enzyme HMG COA reductase. Mol Cytogenet. 2014;7(1):1. doi:10.1186/1755-8166-7-S1-P97

96. Zhang M-Q, Wilkinson B. Drug discovery beyond the 'rule-of-five'. Curr Opin Biotechnol. 2007;18(6):478-488. doi:10.1016/j. copbio.2007.10.005

\section{Publish your work in this journal}

Drug Design, Development and Therapy is an international, peerreviewed open-access journal that spans the spectrum of drug design and development through to clinical applications. Clinical outcomes, patient safety, and programs for the development and effective, safe, and sustained use of medicines are a feature of the journal, which has also been accepted for indexing on PubMed Central. The manuscript management system is completely online and includes a very quick and fair peer-review system, which is all easy to use. Visit http://www. dovepress.com/testimonials.php to read real quotes from published authors. 